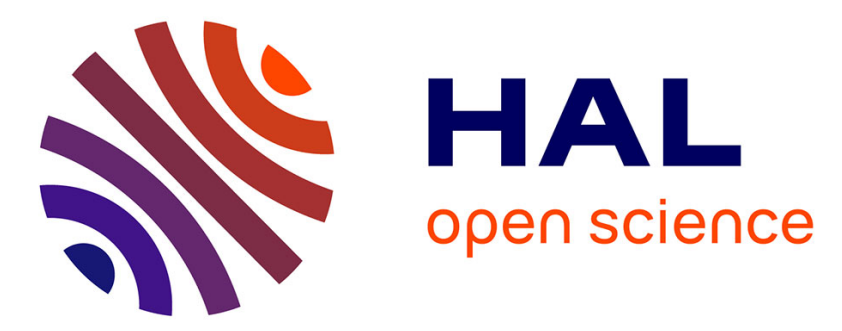

\title{
Eddy current effects in plain and hollow cylinders spinning inside homogeneous magnetic fields: Application to magnetic resonance
}

\author{
G. Aubert, J.-F. Jacquinot, D. Sakellariou
}

\section{- To cite this version:}

G. Aubert, J.-F. Jacquinot, D. Sakellariou. Eddy current effects in plain and hollow cylinders spinning inside homogeneous magnetic fields: Application to magnetic resonance. Journal of Chemical Physics, 2012, 15, pp.154201. 10.1063/1.4756948 . cea-00853763

\section{HAL Id: cea-00853763 https://hal-cea.archives-ouvertes.fr/cea-00853763}

Submitted on 17 May 2019

HAL is a multi-disciplinary open access archive for the deposit and dissemination of scientific research documents, whether they are published or not. The documents may come from teaching and research institutions in France or abroad, or from public or private research centers.
L'archive ouverte pluridisciplinaire HAL, est destinée au dépôt et à la diffusion de documents scientifiques de niveau recherche, publiés ou non, émanant des établissements d'enseignement et de recherche français ou étrangers, des laboratoires publics ou privés. 


\section{Eddy current effects in plain and hollow cylinders spinning inside homogeneous magnetic fields: Application to magnetic resonance}

Cite as: J. Chem. Phys. 137, 154201 (2012); https://doi.org/10.1063/1.4756948

Submitted: 13 July 2012 . Accepted: 18 September 2012 . Published Online: 15 October 2012

G. Aubert, J.-F. Jacquinot, and D. Sakellariou

\section{ARTICLES YOU MAY BE INTERESTED IN}

Torque induced on a conducting cylinder by a slowly rotating magnetic field Journal of Applied Physics 54, 1135 (1983); https://doi.org/10.1063/1.332133

A slowly rotating hollow sphere in a magnetic field: First steps to de-spin a space object American Journal of Physics 84, 181 (2016); https://doi.org/10.1119/1.4936633

Eddy current loss calculation and thermal analysis of axial-flux permanent magnet couplers AIP Advances 7, 025117 (2017); https://doi.org/10.1063/1.4977702 


\title{
Eddy current effects in plain and hollow cylinders spinning inside homogeneous magnetic fields: Application to magnetic resonance
}

\author{
G. Aubert, ${ }^{1}$ J.-F. Jacquinot, ${ }^{2, a)}$ and D. Sakellariou ${ }^{3,4}$ \\ ${ }^{1}$ CEA Saclay, DSM, IRFU, F-91191 Gif-sur-Yvette, France \\ ${ }^{2}$ CEA Saclay, DSM, IRAMIS, SPEC, F-91191 Gif-sur-Yvette, France \\ ${ }^{3}$ CEA Saclay, DSM, IRAMIS, UMR CEA/CNRS no 3299-SIS2M, Laboratoire Structure et Dynamique par \\ Résonance Magnétique, F-91191 Gif-sur-Yvette, France \\ ${ }^{4}$ Département de Chimie, Ecole Normale Supérieure, 24 rue Lhomond, 75231 Paris Cedex 05, France
}

(Received 13 July 2012; accepted 18 September 2012; published online 15 October 2012)

\begin{abstract}
We present a thorough analysis of eddy currents that develop in a rectangular cross section toroid rotating in a uniform magnetic field. The slow rotation regime is assumed. Compact expressions for the current density, the total dissipated power, and the braking torque are given. Examination of the topology of current lines reveals that depending upon the relative dimensions of the side and length of the toroid two different regimes exist. The conditions of existence of the two regimes are analytically established. In view of nuclear magnetic resonance (NMR) applications, we derive the angular variation of the magnetic field created by eddy currents and lay down the formalism necessary for calculating the effect of this field on the NMR spectra of the conductor itself or of a sample corotating with the conductor, a situation encountered when dealing with rotating detectors. Examples of calculations for cases of practical interest are presented. The theory is confronted with available data, and we give guidelines for the design of optimized rotating micro-coils. () 2012 American Institute of Physics. [http://dx.doi.org/10.1063/1.4756948]
\end{abstract}

\section{INTRODUCTION}

When a conductor $\mathrm{C}$ is subject to a magnetic field $\vec{B}_{0}$, a motion of $\mathrm{C}$ or a time dependence of $\vec{B}_{0}$ leads to the generation of Foucault or eddy currents. Such circulating electronic or ionic currents can be used for levitation, for metal detection and identification, for position sensing and structural non-destructive testing, as well as for electromagnetic braking and heating. In this article we treat the specific case of a conductor rotating inside an ultra-uniform magnetic field, like the ones usually present in magnetic resonance (MR) studies. However, the closely related cases of a conductor subject to a rotating magnetic field or subject to an oscillating magnetic field can be similarly treated.

There are several instances where eddy currents can play an important role in nuclear magnetic resonance (NMR): In NMR spectroscopy of conductive samples where the sample must be spun, as in magic angle spinning (MAS), eddy currents can lead to unwanted heating effects. ${ }^{1}$ The influence of the magnetic fields they induce should also be considered. In the recently introduced magic angle coil spinning (MACS) technique, ${ }^{2}$ a tuned coil is spun together with the sample inside a rotor; even if the sample is non-conductive, localized but intense currents can develop inside the coil: they produce heating as well as induced magnetic fields. Heating effects have been reported and a simplified model to account for the experimental findings was described without in-depth analysis ${ }^{3}$ they can be minimized by a good thermal contact with the surface of the rotor, but this does not reduce the effect of induced magnetic fields that should be dealt with by

\footnotetext{
a) Author to whom correspondence should be addressed. Electronic mail: jjacquinot@cea.fr.
}

other means. Rotating detectors have also been introduced in magnetic resonance imaging in order to perform a kind of parallel imaging. ${ }^{4}$ Additionally, the use of eddy current heating has been proposed as a convenient means to vary the temperature of a sample in a NMR rotor inside which electronically conductive parts are judiciously placed. ${ }^{1}$

As a prerequisite for the study of the eddy currents effects in the field of MR we need a quantitative description of these currents and of the fields they produce. Generation of eddy currents resulting from rotation of a conductor has been studied for a variety of conductor shapes: for a sphere, ${ }^{5}$ for a very long cylinder, ${ }^{6}$ and for a very thin disk. ${ }^{7}$ The case of plain and hollow cylinders ${ }^{8,9}$ has also been dealt with. However, the focus in these articles was placed on the power $(\mathrm{P})$ dissipated inside the conductor. No detailed study of the current distribution $(\vec{J})$, or of the magnetic fields generated by eddy currents, has been presented.

Here we give a new treatment that leads to explicit formulae for $\vec{J}$ and $\mathrm{P}$ that are more adapted to a complete study of eddy currents effects. They allow us to establish general important results concerning the topology of the current lines and the symmetry of the fields induced by eddy currents. Useful approximate formulae for particular geometries are derived. Finally, we lay down the formalism necessary for calculating NMR spectra of samples under MAS in the presence of eddy currents effects. We apply our results to discuss eddy current heating in rotating coils, in particular, its variation with the coil dimensions, and give guidelines for the design of optimized rotating micro-coils. We re-examine Foucault heating observations from the literature and finally we predict the effects of the magnetic fields created by eddy currents in NMR experiments involving rotation of a conductive 
sample or rotation of the sample together with the detection coil.

\section{GENERALITIES AND LAYOUT OF THE PROBLEM IN THE SLOW ROTATION REGIME}

We consider a conductor of largest dimension $L$ placed in a uniform magnetic field $\vec{B}_{0}$ and rotating about axis $\vec{z}$ at angular velocity $\omega_{r}=2 \pi v_{r}$. The angular velocity vector is $\vec{\Omega}_{r}=\omega_{r} \vec{u}_{z}$, where $\vec{u}_{z}$ is the unit vector along $\vec{z}$.The material has an isotropic conductivity $\sigma$, and is assumed non-magnetic $\left(\mu=\mu_{0}\right)$. Eddy currents originate from the fact that a free carrier of charge q with local velocity $\vec{v}$ experiences a Lorentz force $q \vec{v} \times \vec{B}_{0}$. This is equivalent to an internal electromotive field $\vec{E}^{\prime}=\vec{v} \times \vec{B}_{0}$ which drives eddy currents circulation inside the conductor. As shown below the field $\vec{E}^{\prime}$, which we term the exciting field, appears naturally when transforming to a frame rotating with the conductor. Two factors are important to determine how eddy currents develop and distribute themselves inside the conductor:

- The orientation of $\vec{E}^{\prime}$ at the bounding surface of the conductor; if $\vec{E}^{\prime}$ has a non-zero normal component, one may anticipate that surface charges will appear and create an extra field $\vec{E}_{c h}^{\prime}$ that adds to $\vec{E}^{\prime}$ and tends to cancel the current flowing perpendicularly to the surface.

- The value of the rotation period $\mathrm{T}=2 \pi / \omega_{\mathrm{r}}$, which should be compared to the characteristic times $\tau_{1}, \tau_{2}$, and $\tau_{3}$, defined below, in order to define the regime to which the problem belongs and treat it at the correct level of approximation.

\section{A. Time constants and rotation regimes}

The first time constant to consider, $\tau_{1}=\mathrm{L} / \mathrm{c}$, is the propagation time of an electromagnetic wave over the distance $\mathrm{L}$.

Time $\tau_{2}$ is the equilibration time of the volumic and surface charges in the conductor. By combining Maxwell's equation $\vec{\nabla} \cdot \vec{E}=\rho / \varepsilon_{0}$ with Ohm's law, $\vec{J}=\sigma \vec{E}$, and the equation for charge conservation, $\vec{\nabla} \cdot \vec{J}=-\frac{d \rho}{d t}$, one obtains

$$
\frac{d \rho}{d t}+\frac{\sigma}{\varepsilon_{0}} \rho=0 .
$$

This equation indicates that no volumic charges exist at equilibrium, and that equilibrium is reached in a time of the order of $\tau_{2}=\varepsilon_{0} / \sigma$.

Time $\tau_{3}$ is the threshold value of the rotation period below which eddy currents become so strong that the fields they create are not negligible compared to the applied field $\vec{B}_{0}$ : the value of $\tau_{3}$ can be estimated by using the fact that according to Lenz' law eddy currents prevent penetration of the magnetic field in a direction perpendicular to the rotation axis. As in the case of rf penetration this effect occurs over a distance $\delta$, the so called skin depth, related to the period $\mathrm{T}$ by $\delta=\sqrt{2 /\left(\mu \sigma \omega_{r}\right)}=\sqrt{\mathrm{T} /(\pi \mu \sigma)}$. Time $\tau_{3}$ corresponds to the period for which $\delta$ is of the order of the characteristic dimension $\mathrm{L}_{\perp}$ of the conductor perpendicular to the rotation axis. We thus have $\tau_{3} \approx \pi \mu \sigma \mathrm{L}_{\perp}^{2}$.
The regime is said to be quasi-static if $T \gg \tau_{1}$. Under this condition, the electric and magnetic fields $\vec{B}$ and $\vec{E}$ at a given time $t$ can be determined from the distribution of charges and currents at the same time t.

The regime is said to be quasi-stationary if $\mathrm{T} \gg \tau_{1}, \tau_{2}$. The surface charges distribution and the currents have time to reach their equilibrium values at each angle during rotation under the influence of the fields present inside the conductor.

The slow rotation regime is defined by the condition

$$
\mathrm{T} \gg \tau_{1}, \tau_{2}, \tau_{3} \quad \text { or equivalently: } \mathrm{T} \gg \tau_{1}, \tau_{2} \text { and } \delta \gg \mathrm{L}_{\perp} \text {, }
$$

which we assume valid from now on. It introduces considerable simplification since the value of the magnetic field inside the conductor can be taken equal to $\vec{B}_{0}$ to a good approximation. It is most appropriate for the determination of eddy currents to use a frame rotating with the conductor. In this frame we can use Ohm's law to derive the current density from the electric fields present in the conductor.

A numerical application with the following values will prove useful in the later discussion. For $\mathrm{L}=10 \mathrm{~mm}, \sigma_{\mathrm{Cu}}$ $=6 \times 10^{7} \mathrm{~S} / \mathrm{m}$, and $\mu=\mu_{0}$, we find $\tau_{1}=3.3 \times 10^{-11} \mathrm{~s}$ and $\tau_{2}=1.5 \times 10^{-19} \mathrm{~s}$. For $\mathrm{L}_{\perp}$ equal to $5 \mathrm{~mm}$ and $100 \mu \mathrm{m}$, we obtain $\tau_{3}=5.9 \mathrm{~ms}$ and $\tau_{3}=2.4 \mu \mathrm{s}$, respectively. $\tau_{1}$ and $\tau_{2}$ are always very short for practical purposes and only $\tau_{3}$ can be comparable to the spinning period.

\section{B. Electric field in the rotating frame}

Taking the origin of coordinates on the axis of rotation, each point $\vec{r}$ of the conductor has an instantaneous velocity $\vec{v}$ :

$$
\vec{v}=\vec{\Omega}_{r} \times \vec{r} .
$$

Assuming $|\vec{v}| \ll \mathrm{c}$ in all the conductor, the electric field $\vec{E}=0$ in the laboratory frame is transformed in the rotating frame into ${ }^{10}$

$$
\vec{E}^{\prime} \approx \vec{E}+\vec{v} \times \vec{B}_{0}=\vec{v} \times \vec{B}_{0} .
$$

From the previous discussion (Secs. II A and II B) we can describe qualitatively in the rotating frame the steps of eddy currents generation. Under the influence of field $\vec{E}^{\prime}$ eddy currents start to develop; except for particular geometries of the conductor, $\vec{E}^{\prime}$ has non-zero components perpendicular to the surface(s) of the conductor so that surface charges appear with a time constant of the order of $\tau_{2}$. They create an additional field $\vec{E}_{c h}^{\prime}$, which adds to $\vec{E}^{\prime}$, modifies the current trajectories, and prevents them from escaping from the conductor. If condition $\tau_{2} \ll \mathrm{T}$ is fulfilled, the fields and charge distributions do not change appreciably on time scale $\tau_{2} \ll \mathrm{t} \ll \mathrm{T}$. On this time scale, we are faced with a electrostatic problem, which is to find the electric field $\vec{E}_{c h}^{\prime}$.

\section{Alternative choices for the electric field in the rotating frame}

Equation (2) for the exciting field leads to the correct value of the eddy currents and surface charges distributions. Since we are not interested in the latter we can choose another field expression that gives the same current distribution: this 
will be the case if we superimpose on $\vec{E}^{\prime}$ an arbitrary gradient $\vec{E}^{\prime \prime}=\vec{\nabla} f(\vec{r})$. Such a field induces additional unphysical surface charges on a time scale $\tau_{2}$, but it does not produce eddy currents since it is not associated with an electromotive field: indeed, the circulation of $\vec{E}^{\prime \prime}$ on any closed loop is zero. The field produced by the additional charges will thus cancel $\vec{E}^{\prime \prime}$. The actual surface charges at the boundaries of the conductor remain, however, important for the calculation of the potentials at the surface of the conductor.

\section{General scheme for the determination of $\vec{E}_{c h}^{\prime}$ and of the current distribution}

The current flowing inside the conductor is induced by the resultant of the exciting field $\vec{E}^{\prime}$ (complemented, if convenient, by an additional field $\vec{E}^{\prime \prime}$ of the form $\vec{E}^{\prime \prime}=\vec{\nabla} \cdot f(\vec{r})$ ) and of the field $\vec{E}_{c h}^{\prime}$ created by surface charges. The latter derives from a potential, which is the solution of Laplace's equation with the boundary condition that the normal component of the total electric field $\vec{E}^{\prime}+\vec{E}_{c h}^{\prime}$ is zero on the surface of the conductor. Once $\vec{E}_{c h}^{\prime}$ has been determined, the current $\vec{J}$ is deduced by application of Ohm's law:

$$
\vec{J}=\sigma\left(\vec{E}^{\prime}+\vec{E}_{c h}^{\prime}\right)
$$

\section{E. Stationarity of the eddy currents distribution in the case of a cylinder}

Although the problem is of electrostatic nature only in the rotating frame, we may note that the exciting field $\vec{E}^{\prime}$ (see Eq. (2)) viewed from the laboratory frame is stationary. The same is true for the boundary condition if the conductor has cylindrical symmetry about the $\mathrm{z}$ axis. The solution of the electrostatic problem and, in particular, the current distribution in this case will thus appear static in the laboratory frame.

\section{CURRENT DISTRIBUTION IN THE CASE OF A HOLLOW CYLINDER}

From now on, we restrict ourselves to the case of a conductor shaped as a toroid of rectangular cross section. It is spun about its axis of symmetry $\vec{z}$ in the presence of a static magnetic field $\vec{B}_{0}$ applied at an angle $\theta$ of $\vec{z}$ (see Fig. 1). The slow rotation regime (Eq. (1)) is assumed. The dimensions of the conductor are: internal radius $a_{1}$, external radius $a_{2}$, and length 2 b. As shown in Fig. 1 we use at a given time two instantaneous Cartesian and cylindrical sets of coordinates $\mathrm{x}, \mathrm{y}$, $\mathrm{z}$ and $\rho, \varphi, \mathrm{z}$ with the origin placed on the plane of symmetry of the conductor. $\vec{u}_{\mathrm{x}}, \vec{u}_{\mathrm{y}}, \vec{u}_{\mathrm{z}}$ and $\vec{u}_{\rho}, \vec{u}_{\varphi}, \vec{u}_{\mathrm{z}}$ denote the unit vectors of the two coordinate systems. The direction of $\vec{y}$ is chosen such that $\vec{B}_{0}$ lies in the yz plane.

Because the equations are linear, we can examine separately the effects of the components of $\vec{B}_{0}$ parallel and perpendicular to $\vec{z}$. Let

$$
\vec{B}_{0}=B_{0} \cos (\theta) \vec{u}_{z}+B_{0} \sin (\theta) \vec{u}_{y} .
$$

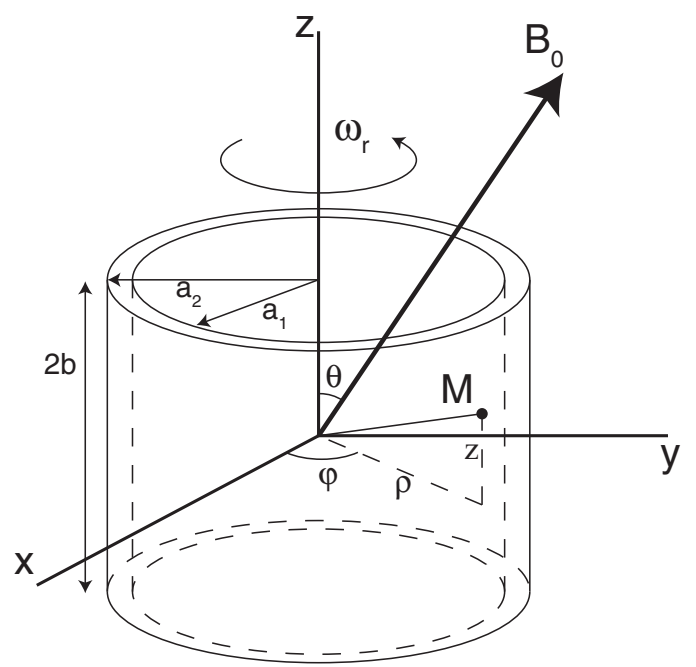

FIG. 1. Sketch of the hollow cylinder rotating inside a magnetic field $\vec{B}_{0}$. The Cartesian frame and cylindrical coordinates used in the calculation are also shown.

The first term produces in the rotating frame an electric field:

$$
\vec{E}^{\prime}=\cos (\theta) \vec{v} \times \vec{B}_{0}=\cos (\theta)\left(\vec{\Omega}_{r} \times \vec{r}\right) \times \vec{B}_{0}=\cos (\theta) \omega_{r} B_{0} \rho \vec{u}_{\rho} .
$$

This field is radial. By a mechanism similar to the Hall effect, charges of opposite sign develop on time scale $\tau \sim \tau_{2}$ on the inner and outer surfaces of the conductor; the electric field they create cancels field $E^{\prime}$ and no eddy currents are induced in the conductor after the equilibration period. We notice that in this case $\vec{E}^{\prime} \propto \vec{\nabla}\left(B_{0} \omega_{r} \rho^{2}\right)$, and as seen in Sec. II C, such a field cannot create eddy currents; this property is independent of the cylindrical shape assumed for the conductor.

From now on, we consider only the perpendicular component (second term) that produces in the rotating frame an electric field:

$$
\vec{E}^{\prime}=-B_{0} \omega_{r} \sin (\theta) y \vec{u}_{z} .
$$

We can also use the alternative form (see Sec. II C)

$$
\begin{aligned}
\vec{E}^{\prime} & =-B_{0} \omega_{r} \sin (\theta) y \vec{u}_{z}+\vec{\nabla} \cdot\left(B_{0} \omega_{r} \sin (\theta) y z\right) \\
& =B_{0} \omega_{r} \sin (\theta) z \vec{u}_{y} .
\end{aligned}
$$

Equation (3a) has been used in Refs. 8 and 9. We found that Eq. (3b) leads to more compact and tractable formulae that enabled us to make a thorough study of the eddy currents and related effects; we shall thus use this form from now on.

We define the reduced fields $\vec{e}^{\prime}=\vec{E}^{\prime} /\left(B_{0} \omega_{r} \sin (\theta)\right)$ and $\vec{e}_{c h}^{\prime}=\vec{E}_{c h}^{\prime} /\left(B_{0} \omega_{r} \sin (\theta)\right)$. In cylindrical coordinates

$$
\vec{e}^{\prime}=z \sin (\varphi) \vec{u}_{\rho}+z \cos (\varphi) \vec{u}_{\varphi}
$$

Our task is to find $\vec{e}_{c h}^{\prime}$. It derives from a potential $v_{c h}^{\prime}$ which obeys Laplace's equation with the boundary condition that the normal component of $\vec{e}^{\prime}+\vec{e}_{c h}^{\prime}$ is zero at the surface of the conductor.

$v_{c h}^{\prime}$ can be decomposed as the sum of two solutions $v_{c h 1}^{\prime}$ and $v_{c h 2}^{\prime}$. $v_{c h 1}^{\prime}$ is obtained with the following boundary 
conditions (BC's):

$$
\begin{aligned}
& \frac{\partial v_{c h 1}^{\prime}}{\partial \rho}=0, \rho=a_{1}, \forall z \in[-b, b], \forall \varphi \in[0,2 \pi], \\
& \frac{\partial v_{c h 1}^{\prime}}{\partial \rho}=z \sin (\varphi), \rho=a_{2}, \forall z \in[-b, b], \forall \varphi \in[0,2 \pi], \\
& \frac{\partial v_{c h 1}^{\prime}}{\partial z}=0, z= \pm b, \forall \rho \in\left[a_{1}, a_{2}\right], \forall \varphi \in[0,2 \pi] .
\end{aligned}
$$

The second solution is obtained by permuting $\mathrm{a}_{1}$ and $\mathrm{a}_{2}$.

The general method for solving Laplace's equation in cylindrical coordinates is given in standard textbooks ${ }^{11,12}$ It relies on variable separation: one looks for solutions that can be written as the product $\mathrm{R}(\rho) \mathrm{Q}(\varphi) \mathrm{Z}(\mathrm{z})$ of functions of $\rho, \varphi$, $\mathrm{z}$, for which three differential equations are obtained; here we note that (BC2) imposes that $\mathrm{Q}(\varphi)=\sin (\varphi)$ and that $\mathrm{Z}(\mathrm{z})$ is an odd function of $\mathrm{z}$. We may choose $\mathrm{Z}(\mathrm{z})=\sin (\mathrm{mz})$ or $\mathrm{Z}(\mathrm{z})$ $=\sinh \left(\mathrm{m}^{\prime} \mathrm{z}\right)$ where $\mathrm{m}$ and $\mathrm{m}^{\prime}$ are non-integer indexation parameters to be determined from the boundary conditions; the first choice leads to a solution for our problem of the form

$$
v_{c h 1}^{\prime}=\sum_{m}\left[A_{1 m} I_{1}(m \rho)+B_{1 m} K_{1}(m \rho)\right] \sin (m z) \sin (\varphi),
$$

where $I_{1}$ and $K_{1}$ are modified Bessel functions of first order and

$$
m=\frac{(2 n+1) \pi}{2 b}, n=0,1, \ldots,
$$

which ensures that $(\mathrm{BC} 3)$ is verified; (BC1) immediately gives for each value of $\mathrm{m}$

$$
A_{1 m}\left[I_{0}\left(m a_{1}\right)+I_{2}\left(m a_{1}\right)\right]-B_{1 m}\left[K_{0}\left(m a_{1}\right)+K_{2}\left(m a_{1}\right)\right]=0 .
$$

Equation (BC2) gives

$$
\begin{gathered}
\sum_{m} \frac{m}{2}\left\{A_{1 m}\left[I_{0}\left(m a_{2}\right)+I_{2}\left(m a_{2}\right)\right]-B_{1 m}\left[K_{0}\left(m a_{2}\right)\right.\right. \\
\left.\left.+K_{2}\left(m a_{2}\right)\right]\right\} \sin (m z)=z, \forall z \in[-b, b] .
\end{gathered}
$$

By identification with the Fourier development of $\mathrm{z}$ :

$$
z=\frac{8 b}{\pi^{2}} \sum_{n=0}^{\infty} \frac{(-1)^{n}}{(2 n+1)^{2}} \sin \left[(2 n+1) \frac{\pi}{2} \frac{z}{b}\right]
$$

one obtains for each value of $m$

$$
\begin{aligned}
& A_{1 m}\left[I_{0}\left(m a_{2}\right)+I_{2}\left(m a_{2}\right)\right]-B_{1 m}\left[K_{0}\left(m a_{2}\right)+K_{2}\left(m a_{2}\right)\right] \\
& =(-1)^{n} \frac{4}{m^{3} b} .
\end{aligned}
$$

By solving Eqs. (4) and (5) for $\mathrm{A}_{1 \mathrm{~m}}$ and $\mathrm{B}_{1 \mathrm{~m}}$ one obtains the solution for $v_{c h 1}^{\prime}$. The corresponding solution for $v_{c h 2}^{\prime}$ is obtained by permutation of $\mathrm{a}_{1}$ and $\mathrm{a}_{2}$. The final result for $v_{c h}^{\prime}$ is

$$
v_{c h}^{\prime}=\sum_{m}\left[A_{m} I_{1}(m \rho)+B_{m} K_{1}(m \rho)\right] \sin (m z) \sin (\varphi)
$$

with

$$
\begin{aligned}
A_{m} & =(-1)^{n+1} \frac{4}{b m^{3}} \frac{K_{0}\left(m a_{2}\right)+K_{2}\left(m a_{2}\right)-\left[K_{0}\left(m a_{1}\right)+K_{2}\left(m a_{1}\right)\right]}{\left[I_{0}\left(m a_{2}\right)+I_{2}\left(m a_{2}\right)\right]\left[K_{0}\left(m a_{1}\right)+K_{2}\left(m a_{1}\right)\right]-\left[I_{0}\left(m a_{1}\right)+I_{2}\left(m a_{1}\right)\right]\left[K_{0}\left(m a_{2}\right)+K_{2}\left(m a_{2}\right)\right]}, \\
B_{m} & =(-1)^{n+1} \frac{4}{b m^{3}} \frac{I_{0}\left(m a_{2}\right)+I_{2}\left(m a_{2}\right)-\left[I_{0}\left(m a_{1}\right)+I_{2}\left(m a_{1}\right)\right]}{\left[I_{0}\left(m a_{2}\right)+I_{2}\left(m a_{2}\right)\right]\left[K_{0}\left(m a_{1}\right)+K_{2}\left(m a_{1}\right)\right]-\left[I_{0}\left(m a_{1}\right)+I_{2}\left(m a_{1}\right)\right]\left[K_{0}\left(m a_{2}\right)+K_{2}\left(m a_{2}\right)\right]},
\end{aligned}
$$

and

$$
m=\frac{(2 n+1) \pi}{2 b}, n=0,1, \ldots
$$

$$
j_{z}=\left\{-\sum_{m} m\left[A_{m} I_{1}(m \rho)+B_{m} K_{1}(m \rho)\right] \cos (m z)\right\} \sin (\varphi) .
$$

Defining:

$$
\vec{j}=\vec{J} / \sigma B_{0} \omega_{r} \sin (\theta),
$$

Ohm's law gives $\vec{j}=\vec{e}^{\prime}-\vec{\nabla} v_{c h}^{\prime}$, whence we obtain for the three components of $\vec{j}$

$$
\begin{aligned}
j_{\rho}= & \left\{z-\frac{1}{2} \sum_{m} m\left\{A_{m}\left[I_{0}(m \rho)+I_{2}(m \rho)\right]\right.\right. \\
& \left.\left.-B_{m}\left[K_{0}(m \rho)+K_{2}(m \rho)\right]\right\} \sin (m z)\right\} \sin (\varphi), \\
j_{\varphi}= & \left\{z-\frac{1}{\rho} \sum_{m}\left[A_{m} I_{1}(m \rho)+B_{m} K_{1}(m \rho)\right] \sin (m z)\right\} \cos (\varphi),
\end{aligned}
$$

The three components of $\vec{j}$ have much simpler expressions in the case of a plain cylinder $\left(a_{1}=0\right)$. They are obtained by substituting in Eqs. (8a)-(8c) $B_{m}=0$ and

$$
A_{m}=(-1)^{n} \frac{4}{b m^{3}} \frac{1}{\left[I_{0}\left(m a_{2}\right)+I_{2}\left(m a_{2}\right)\right]} .
$$

\section{A. Symmetry of the solution}

Inspection of Eqs. (8a)-(8c) reveals how symmetry operations with respect to the three planes $\mathrm{xOy}, \mathrm{xOz}$, and $\mathrm{yOz}$ 
affect the three components of $\vec{J}$, namely:

$$
\begin{gathered}
x \rightarrow-x \leftrightarrow J_{x} \rightarrow-J_{x}, J_{y} \rightarrow J_{y}, J_{z} \rightarrow J_{z}, \\
y \rightarrow-y \leftrightarrow J_{x} \rightarrow-J_{x}, J_{y} \rightarrow J_{y}, J_{z} \rightarrow-J_{z}, \\
z \rightarrow-z \leftrightarrow J_{x} \rightarrow-J_{x}, J_{y} \rightarrow-J_{y}, J_{z} \rightarrow J_{z} .
\end{gathered}
$$

\section{CALCULATION OF THE EDDY CURRENT LOSSES $P$ AND OF THE BRAKING TORQUE $\Gamma_{Z}$}

The power dissipation P can be calculated from Joule's formula, which according to our notation (Eq. (7)) and to the symmetry of the current distribution $\vec{J}$, can be written

$P=8 \sigma\left(B_{0} \omega_{r} \sin (\theta)\right)^{2} \int_{a_{1}}^{a_{2}} \int_{0}^{\pi / 2} \int_{0}^{b}\left(j_{\rho}^{2}+j_{\varphi}^{2}+j_{z}^{2}\right)^{2} \rho d \rho d \varphi d z$.

The calculation is possible (see supplemental material ${ }^{13}$ ) but complex since the function to integrate is quadratic in $\vec{J}$; furthermore, it does not lead to a simple formula. It is much simpler to use the relation $P=-\omega_{r} \Gamma_{z}$ between the power $\mathrm{P}$ and the braking torque $\Gamma_{z}=\vec{\Gamma} \cdot \vec{u}_{z}$, whose direction points along the rotation axis $\vec{z}$. The general expression for $\vec{\Gamma}$ is: $\vec{\Gamma}=\iiint_{V} \vec{r} \times \vec{F} d v$, where $\vec{F} d v=\vec{J} \times \vec{B}_{0} d v$ is Laplace's force acting on volume $d v$. The contribution to $\Gamma_{\mathrm{z}}$ of the parallel component $\mathrm{B}_{0 z}$ of $\vec{B}_{0}$ is the sum of $-B_{0 z} \iiint_{V} x J_{x} d v$ and $-B_{0 z} \iiint_{V} y J_{y} d v$. These two terms are zero by symmetry (see (III A)). We just need to consider the contribution of the perpendicular component $\mathrm{B}_{0 \mathrm{x}}=\mathrm{B}_{0} \sin (\theta)$ which is found equal to

$$
\Gamma_{z}=B_{0} \sin (\theta) \iiint_{V} y J_{z}(x, y, z) d v
$$

or in cylindrical coordinates

$$
\Gamma_{z}=8 B_{0}^{2} \omega_{r} \sigma \sin ^{2}(\theta) \int_{a_{1}}^{a_{2}} \int_{0}^{\pi / 2} \int_{0}^{b} j_{z} \rho^{2} \sin (\varphi) d z d \varphi d \rho,
$$

where use has been made of Eq. (7) and of the symmetry of the current distribution. The function to integrate is linear in $\mathrm{j}_{\mathrm{z}}$. Substitution of $\mathrm{j}_{\mathrm{z}}$ by its value given by Eq. (8c) leads to

$$
\begin{aligned}
\Gamma_{z}= & -8 B_{0}^{2} \omega_{r} \sigma \sin ^{2}(\theta) \sum_{m} \int_{a_{1}}^{a_{2}} \int_{0}^{\pi / 2} \int_{0}^{b} m\left[A_{m} I_{1}(m \rho)\right. \\
& \left.+B_{m} K_{1}(m \rho)\right] \rho^{2} \cos (m z) \sin ^{2}(\varphi) d z d \varphi d \rho .
\end{aligned}
$$

Using $\int_{0}^{\pi / 2} \sin ^{2}(\varphi) d \varphi=\pi / 4$ and $\int_{0}^{b} \cos (m z) d z=\frac{(-1)^{n}}{m}$, we get

$$
\begin{aligned}
\Gamma_{z}= & -2 \pi B_{0}^{2} \omega_{r} \sigma \sin ^{2}(\theta) \sum_{n}(-1)^{n} \int_{a_{1}}^{a_{2}} \rho^{2}\left[A_{m} I_{1}(m \rho)\right. \\
& \left.+B_{m} K_{1}(m \rho)\right] d \rho .
\end{aligned}
$$

For the last integration we use $\int u^{2} I_{1}(u) d u=u^{2} I_{2}(u)$ and $\int u^{2} K_{1}(u) d u=-u^{2} K_{2}(u)$ and get

$$
\begin{aligned}
P= & -\omega_{r} \Gamma_{z}=2 \pi B_{0}^{2} \omega_{r}^{2} \sigma \sin ^{2}(\theta) \sum_{n}(-1)^{n} \\
& \times\left|\frac{\rho^{2}}{m}\left[A_{m} I_{2}(m \rho)-B_{m} K_{2}(m \rho)\right]\right|_{a_{1}}^{a_{2}}
\end{aligned}
$$

with $m=\frac{(2 n+1) \pi}{2 b}, n=0,1, \ldots$ (the notation $|f(x)|_{a}^{b}$ stands for $\mathrm{f}(\mathrm{b})-\mathrm{f}(\mathrm{a}))$.

Case of a plain cylinder: The formula for a plain cylin$\operatorname{der}\left(a_{1}=0\right)$ is obtained from Eq. (11) taking $A_{m}$ as given by Eq. (9), $\mathrm{B}_{\mathrm{m}}=0$, and dropping out the $\rho=\mathrm{a}_{1}$ contribution in the $\|_{\rho=a_{1}}^{\rho=a_{2}}$ term. One obtains in this case

$$
\begin{aligned}
P= & \frac{128}{\pi^{3}} B_{0}^{2} \omega_{r}^{2} \sigma \sin ^{2}(\theta) a_{2}^{2} b^{3} \\
& \times \sum_{n} \frac{1}{(2 n+1)^{4}} \frac{I_{2}\left(m a_{2}\right)}{I_{0}\left(m a_{2}\right)+I_{2}\left(m a_{2}\right)} .
\end{aligned}
$$

\section{A. Variation of $\mathbf{P}$ with shape dimensions}

We have studied the variation of $\mathrm{P}$ with cylinder dimensions for two geometries, which have been previously studied in Ref. 8. The comparison is shown in Fig. 2: the left part corresponds to a plain cylinder of radius $\mathrm{a}_{2}$ and the right part to a hollow cylinder with $\mathrm{b} / \mathrm{a}_{2}=1$. Although our formulae are quite different from those of Ref. 8, the results are in perfect agreement.

\section{B. Case of a small cross section toroid}

Let

$$
\begin{aligned}
& a_{1}=(1-\varepsilon / 2) a, \\
& a_{2}=(1+\varepsilon / 2) a, \\
& b=\beta a
\end{aligned}
$$

with $\varepsilon \ll 1, \beta \ll 1$.

We obtain an approximate value of $\mathrm{P}$ by developing $\mathrm{P}$ in $\varepsilon$ and $\beta$. The starting point of the development is an alternative formula for $\mathrm{P}$ demonstrated in supplemental material. ${ }^{13}$ The

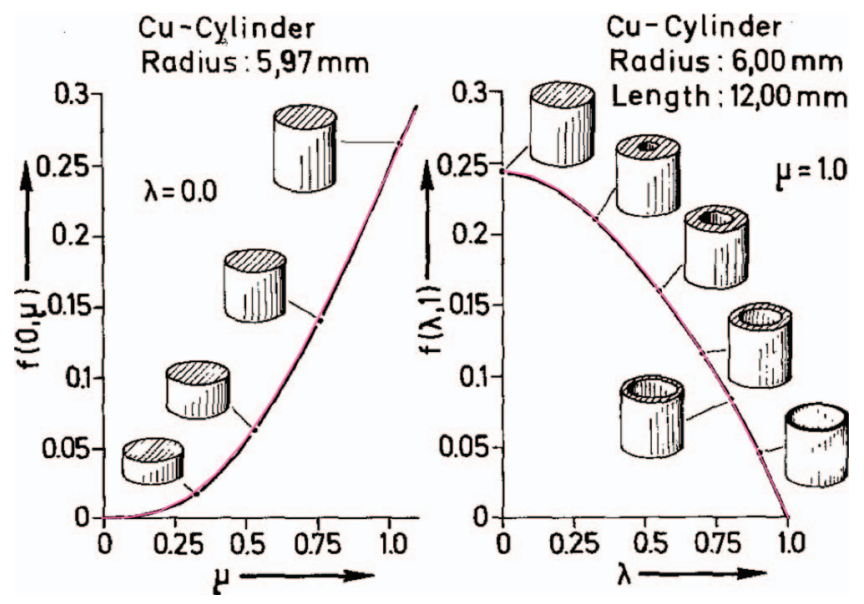

FIG. 2. Comparison of our results (red curves) with those of Ref. 8 (black curves) for a plain cylinder (left) and a hollow one (right). The figure from Ref. 8 was scanned and used as the background on the top of which we plotted our data on the same scale. The coincidence is almost perfect. The power $\mathrm{P}$ was expressed as $P=\pi \sigma\left(B_{0} \omega_{r} \sin \theta\right)^{2} a_{2}^{5} f(\lambda, \mu)$ with $\lambda=a_{1} / a_{2}, \mu=b / a_{2}$, and $f(\lambda, \mu)$ being plotted. Reprinted with permission from R. Schäfer and C. Heiden, Appl. Phys. 9, 121-125 (1976). Copyright 1976 Springer Science and Business Media. 
result to lowest order is

$$
\begin{aligned}
\frac{P}{\sigma\left(B_{0} \omega_{r} \sin (\theta)\right)^{2}} & =\frac{4 \pi}{3} a^{5} \beta^{3} \varepsilon-8 \pi a^{5} \beta^{4} \sum_{k} \frac{1}{k^{5}} \tanh \left(\frac{1}{2} k \frac{\varepsilon}{\beta}\right), \\
k & =(2 n+1) \frac{\pi}{2}, n=0,1, \ldots
\end{aligned}
$$

For arbitrary values of the ratio $\beta / \varepsilon, \mathrm{P}$ does not follow a power law $P \propto a^{5} \varepsilon^{u} \beta^{v}$. It is easily shown that if $\beta / \varepsilon \gg 1$, $P \approx \frac{2 \pi}{3} a^{5} \beta^{3} \varepsilon$ and if $\beta / \varepsilon \ll 1, P \approx \frac{4 \pi}{3} a^{5} \beta^{3} \varepsilon$.

To illustrate the different dependences of $\mathrm{P}$ upon $\alpha$ and $\beta$, we calculated $\mathrm{P}$ according to Eq. (13) when the ratio $\varepsilon / \beta$ departs from the value $\varepsilon_{0} / \beta_{0}=2$ corresponding to a square cross section. The variation of $\mathrm{P}$ is compared to its upper and lower limits $P=\frac{4 \pi}{3} a^{5} \beta^{3} \varepsilon$ (for $\beta / \varepsilon \ll 1$ ) and $P=\frac{2 \pi}{3} a^{5} \beta^{3} \varepsilon$ (for $\beta / \varepsilon \gg 1$ ). It can be seen that although $\mathrm{P}$ does not follow a strict power law variation, the influence of $\beta$ is more critical, and this point should be kept in mind for the design of coils of rectangular cross section. Experimental data from lithographically manufactured coils would be highly valuable to confirm these predictions, which are important for the design of optimized spinning micro-coils.

\section{Other particular geometries}

We give in Appendix A formulae valid for cylinders having one of their dimensions infinitely large or small. This is the case for infinitely long tubes, infinitely thin tubes of finite length, and infinitely thin disks with or without a concentric hole. We also give for the case examined in Sec. IV B a more extended development of $\mathrm{P}$.

\section{Two examples taken from the literature in the field of NMR}

\section{Aguiar et al.}

These authors ${ }^{3}$ have used finite elements calculations to evaluate eddy currents losses in rotating micro-coils. Their starting point was the calculation of losses inside a rotating circular loop of rectangular cross section. The result was then extended with some approximation to helicoidal coils and with a further approximation to helicoidal coils made of a wire of circular cross section.

The model presented here allows checking the validity of their initial numerical calculation. This was done for a loop rotating at magic angle at frequency $v_{\mathrm{r}}=15 \mathrm{kHz}$ in a field $\mathrm{B}_{0}$ $=11.7 \mathrm{~T}$. The loop has an average radius $\mathrm{a}=\left(\mathrm{a}_{1}+\mathrm{a}_{2}\right) / 2$ $=0.35 \mathrm{~mm}$ and a square cross section of side $\mathrm{a}_{2}-\mathrm{a}_{1}$ $=2 \mathrm{~b}=25 \mu \mathrm{m}$. We find the same result $\mathrm{P}=2.4 \mathrm{~mW}$ with the fem software Flux $3 \mathrm{~d},{ }^{14}$ with the analytical expression (11) and with the approximate expression (13).

In Ref. 3 the authors empirically fitted the variation of $P$ for a limited set of values of $a, a_{2}-a_{1}$, and $b$, with $\left(a_{2}-a_{1}\right) / b$ centred on the value 2 and found a power law with non-integer exponents. A similar result is found from the calculation displayed in Fig. 3 which indicates $P \propto \alpha^{1.3} \beta^{2.7}$ in the vicinity of $\varepsilon / \beta=2$.

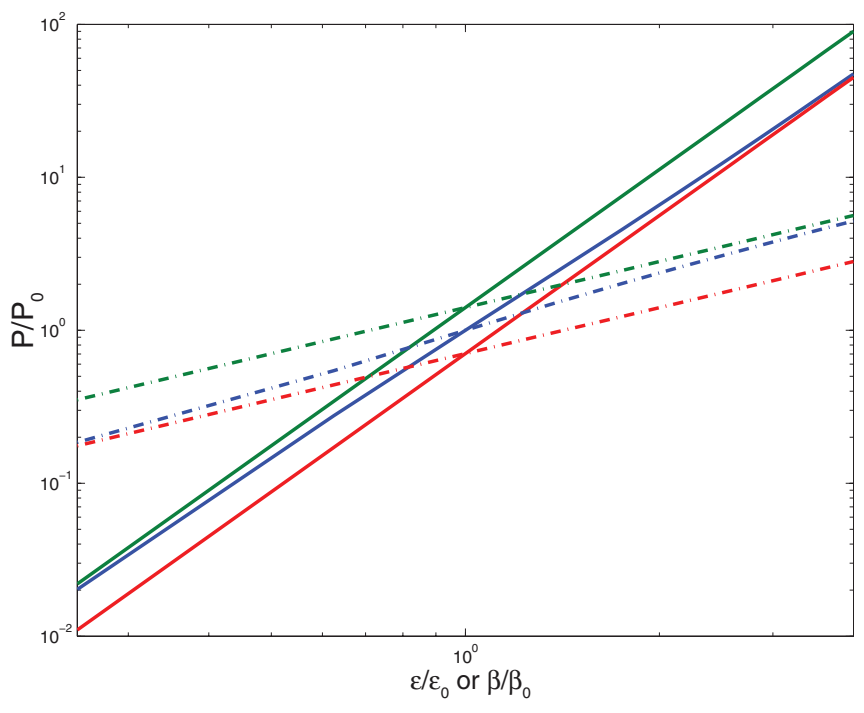

FIG. 3. Variation of the power (P) with side lengths for a small rectangular cross-section toroid as a function of $\varepsilon$ (dashed-dotted curve) or $\beta$ (plain curve); $\varepsilon$ and $\beta$ are related to $\mathrm{b}$ and $\left(\mathrm{a}_{2}-\mathrm{a}_{1}\right)$ by Eq. (12). $\mathrm{P}$ is normalised with respect to its value $\mathrm{P}=\mathrm{P}_{0}$ for $\varepsilon_{0} / \beta_{0}=2$, the value corresponding to a square cross section. The upper and lower limits of $\mathrm{P}$, corresponding to $\varepsilon / \beta \gg 1$ and $\varepsilon / \beta \ll 1$, respectively, are shown as red and green curves.

The slow regime condition was met in this simulation since the skin depth $\delta$ in copper at $v_{r}=15 \mathrm{kHz}$ is equal to $540 \mu \mathrm{m}$, which is much larger than the lateral dimension of the wire.

\section{Yesinowski et al.}

These authors ${ }^{1}$ have observed NMR effects linked to a temperature elevation of their CuI conductive sample in the course of MAS rotation. Their theoretical treatment is based on a simple form of the currents flowing in the cylindrical sample which they take as

$$
\vec{J}(r)=\sigma \vec{v}(r) \times \vec{B}_{0},
$$

where $\vec{v}(r)$ is the velocity at point $r$.

As shown in Sec. II B this amounts to neglecting the surface charges at the ends of the sample. This could possibly be a valid approximation if $\mathrm{T}<\tau_{2}$, since in this case surface charges do not have time to accumulate at the end of the cylinder. For the realistic case $\mathrm{T}>\tau_{2}$, Eq. (14) can only give an approximate value for the case of a long cylinder. For the geometry of sample used in Ref. 1 , diameter $2 \mathrm{a}_{2}=2.9 \mathrm{~mm}$, length $2 b=14 \mathrm{~mm}$, Eq. (14) leads to an overestimation of $\mathrm{P}$ by a factor of 1.13 . We have checked this result by numerical simulation, using the commercial software Flux3d and the values of the experimental parameters of Ref. 1 .

The slow regime condition was met in this experiment because of the low conductivity of the conducting material studied. For a conductivity $\sigma=83 \mathrm{~S} / \mathrm{m}, \delta$ at $\nu_{\mathrm{r}}=10 \mathrm{kHz}$ is equal to $550 \mathrm{~mm}$ which is much larger than the diameter of the sample. 


\section{E. Discussion: Improving performances of rotating micro-coils}

In this section we consider the potential limitations of micro-coils due to eddy current heating. Limitations associated with the fields induced by eddy currents will be examined in Sec. VI.

Two cases are of special interest for improving spinning micro-coils detectors. The first concerns miniaturizing further the detector in order to spin as fast as possible with limited heating. We have seen that micro-solenoids can be manufactured down to $50 \mu \mathrm{m}$ in diameter, using a $10 \mu \mathrm{m}$ diameter wire. ${ }^{15}$ Such micro-coils can be used to detect pico-liter volumes. Using Eq. (11), we can estimate the dissipated power P in an ultra-small micro-coil having the following characteristics: diameter of the coil $=50 \mu \mathrm{m}$, number of turns $=5$, and side of the square cross section copper wire $=10 \mu \mathrm{m}$. We find: $\mathrm{P}=150 \mu \mathrm{W}$ for a coil rotating at magic angle in a field $\mathrm{B}_{0}=11.7 \mathrm{~T}$ at frequency $v_{\mathrm{r}}=40 \mathrm{kHz}$. This estimate is much smaller than the values previously reported ${ }^{2,3}$ and indicates that further miniaturisation would allow very high spinning frequencies.

The second case aims at estimating the heat generated when spinning slowly for sensitive samples like biopsies. Diamagnetic susceptibility broadening effects upon ${ }^{1} \mathrm{H}$ NMR spectra can be averaged even at moderate spinning frequencies. Furthermore, sideband suppression sequences can be successfully applied leading to pure isotropic spectra. ${ }^{16,17} \mathrm{In}$ such cases we consider a micro-solenoid of 5 turns, $500 \mu \mathrm{m}$ coil diameter wound using a $50 \mu \mathrm{m}$ square cross section copper wire and spinning at $200 \mathrm{~Hz}$ oriented at the magic angle inside a $11.7 \mathrm{~T}$ magnet. The estimated power dissipation using Eq. (11) is $\mathrm{P}=50 \mu \mathrm{W}$. This estimate after modelling of the temperature distribution ${ }^{3}$ indicates that, even in the presence of a very poorly thermally conductive insert made of KelF, the increase in sample temperature would not exceed $0.05^{\circ} \mathrm{C}$. Using the relatively large temperature dependence of the water (HDO) chemical shift ${ }^{18}\left(-11.9 \mathrm{ppb} /{ }^{\circ} \mathrm{C}\right)$, one expects that such heating would lead to a maximum ${ }^{1} \mathrm{H}$ frequency shift of $\sim 0.25 \mathrm{~Hz}$ at $500 \mathrm{MHz}$ Larmor frequency. In metabolomics studies this will not be an issue for metabolite identification. Furthermore, the line-width increase due to temperature gradients is not expected to exceed $\sim 20 \%$ of this value.

\section{EDDY CURRENTS TRAJECTORIES}

In this section we examine the eddy current lines patterns in plain and hollow cylinders. We show that for certain aspect ratios $b / a_{2}$, there is a cross-over when $a_{1}$ is varied from the topology characteristic of a plain cylinder to a new topology where two types of lines coexist: one type that extends over one half of the hollow cylinder and another one that is localized in one quarter of the hollow cylinder. The condition of existence of this topology is analytically established.

We begin the discussion by a numerical calculation of the current lines pattern in plain and hollow cylinders. Introducing the curvilinear variable $\mathrm{h}$, a current trajectory starting at point $\rho_{0}, \varphi_{0}, \mathrm{z}_{0}$ can be calculated point by point using the

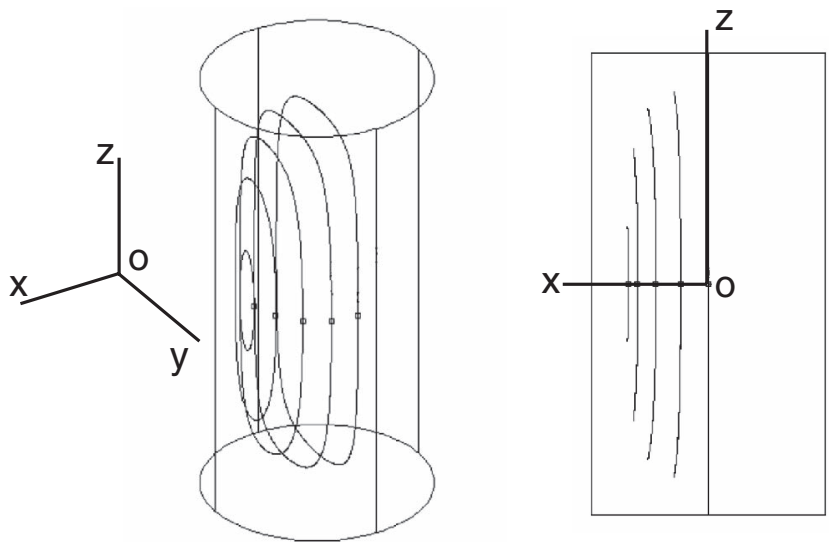

FIG. 4. Current trajectories (presented in the laboratory frame) in a plain cylinder with length/diameter $=2$ seen from two directions. Only half of the trajectories are shown, the other half being obtained from mirror symmetry along the yOz plane. Data obtained using the 3D numerical electromagnetics software Flux3D. The values of the parameters used for the calculation are: a $=1 \mathrm{~mm}, \mathrm{~b}=2 \mathrm{~mm}, \mathrm{~B}_{0}=9.6 \mathrm{~T}, v_{\mathrm{r}}=1 \mathrm{kHz}$, and $\sigma=5.810^{7} \mathrm{~S} / \mathrm{m}$ (copper). The magnetic field is applied perpendicular to the axis of the cylinder, which coincides with the rotation axis. This would correspond to a cylinder spun at the magic angle inside a magnetic field of $\mathrm{B}_{0}=11.7 \mathrm{~T}$. The cylinder and surrounding volume was meshed with 360000 volumic elements.

relation of recurrence:

$$
\begin{aligned}
& \rho_{n+1}=\rho_{n}+h J_{\rho}\left(\rho_{n}, \varphi_{n}, z_{n}\right), \\
& \varphi_{n+1}=\varphi_{n}+h \frac{J_{\varphi}\left(\rho_{n}, \varphi_{n}, z_{n}\right)}{\rho_{n}}, \\
& z_{n+1}=z_{n}+h J_{z}\left(\rho_{n}, \varphi_{n}, z_{n}\right) .
\end{aligned}
$$

This procedure gives the correct trajectory in the limit $\mathrm{h} \rightarrow 0$; better convergence is obtained with higher order "tracking" methods. We have used a fourth order Runge-Kutta algorithm.

\section{A. Case of a plain cylinder}

Figure 4 shows the trajectories in the case of a plain cylinder of radius $a$, with $b / a=2$. They are of oval shape and symmetrical with respect to the xz plane. Except in the yz plane the trajectories are not planar: they bend away from the yz plane in their upper and lower parts. For the two cases $\mathrm{b} / \mathrm{a} \rightarrow 0$ and $\mathrm{b} / \mathrm{a} \rightarrow \infty$ we recover the known results that in the first case the currents trajectories are roughly rectangular with their long sides parallel to the y axis (Ref. 7) and that in the second case the trajectories consist mainly of straight lines parallel to the $\mathrm{z}$ axis (Ref. 6); indeed in this case we can use for $\vec{E}^{\prime}$ Eq. (3a) and neglect surface charges at both ends of the cylinder. Then to a good approximation we have $\vec{J}=-\sigma \vec{B}_{0} \omega_{r} \sin (\theta) y \vec{u}_{z}$. It should be mentioned that the case of trajectories consisting of straight lines parallel to the z-axis is exactly the situation assumed in Ref. 1 corresponding to an infinite cylinder.

\section{B. Case of a hollow cylinder}

The situation is more complex in this case. For some values of $b / a_{2}$ and $a_{1} / a_{2}$ as in Fig. 5(a) the current lines are very similar to those found for a plain cylinder: they are simply "pushed" outwards by the presence of the hole. For other 
(a)

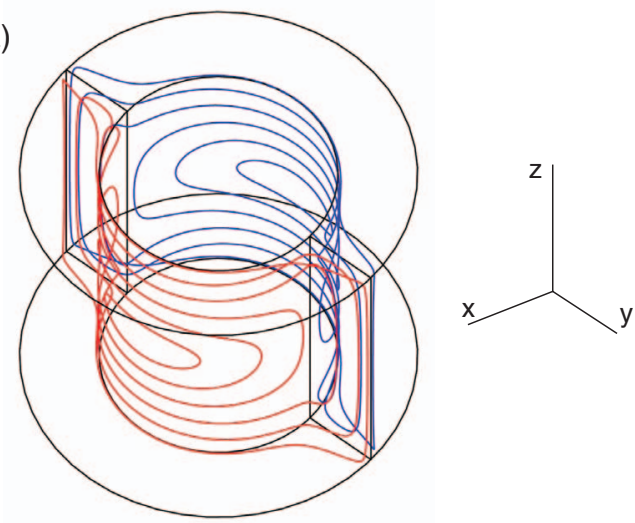

(b)

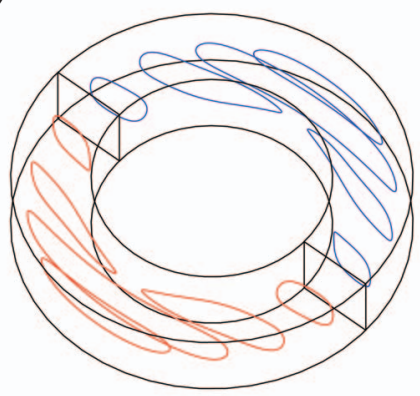

FIG. 5. Current trajectories calculated analytically using Eqs. (8a)-(8c). (a) $\mathrm{a}_{1}=0.75, \mathrm{a}_{2}=1.25, \mathrm{~b}=1$ (a.u.); (b) $\mathrm{a}_{1}=0.75$, $\mathrm{a}_{2}=1.25$, b $=0.25$ (a.u).

values of $b / a_{2}$ and $a_{1} / a_{2}$, as in Fig. 5(b), a new type of trajectory appear: they are located nearby the yz plane and consist of more confined loops that do not cross the xz plane. Each of these loops has a symmetrical counterpart on the other side of the xz plane.

\section{Condition of existence of a 2-type of trajectory topology}

The difference in the two types of behavior represented in Fig. 5 is reflected on the maps of $J_{z}$ in the xy plane, as shown in Fig. 6.

One can see that in case (a), $J_{z}$ has a constant sign in the half-plane $\mathrm{y}>0(\varphi>0)$ and reverses sign for $\mathrm{y}<0(\varphi<0)$. In case (b), there are two regions of positive and negative sign in the half-plane $\mathrm{y}>0$; they are separated by a circular line where $\mathrm{J}_{\mathrm{z}}=0$. From Eq. (8c), condition $\mathrm{J}_{\mathrm{z}}=0$ in the xy plane corresponds to

$$
\sum_{m} m\left[A_{m} I_{1}(m \rho)+B_{m} K_{1}(m \rho)\right]=0 .
$$

The analytical condition of existence for a 2-type of trajectory topology is thus that Eq. (15) has a solution $\rho_{0}$ that satisfies $\mathrm{a}_{1}<\rho_{0}<\mathrm{a}_{2}$. For a given value of $\mathrm{a}_{1} / \mathrm{a}_{2}$ there is

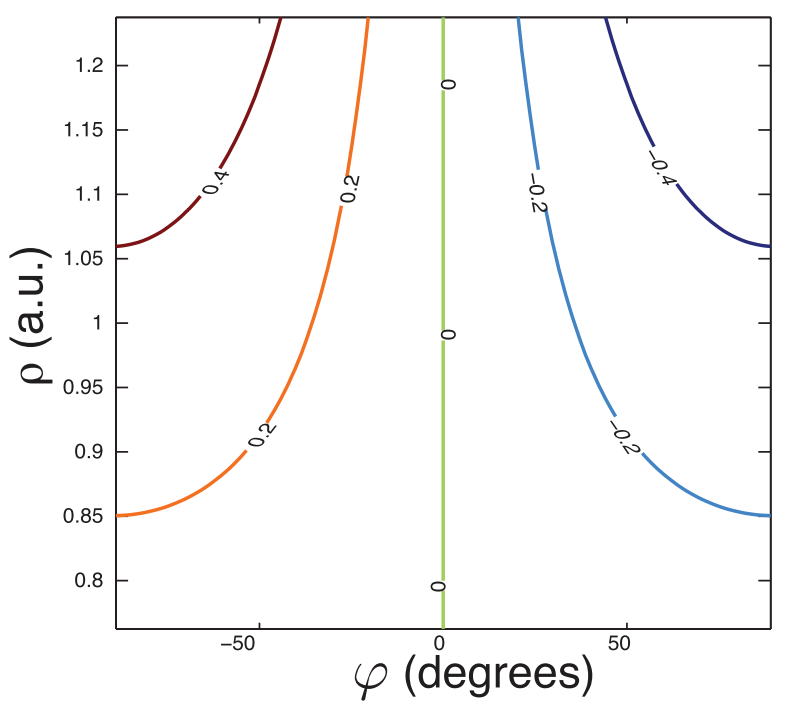

a threshold value $b_{t}$ of $b / a_{2}$ above which only one type of trajectory exists. The variation of $b_{t}$ with $a_{1} / a_{2}$ is shown in Fig. 7. The highest value of $b_{t}$ is $\sim 0.89$; above this value of aspect ratio, a hollow cylinder has the same topology of current lines as a plain cylinder.

\section{FIELDS CREATED BY EDDY CURRENTS}

As already mentioned, in a cylindrical conductor eddy currents are stationary. They produce a static field $\vec{B}_{f}$, which can modify the NMR spectrum. We thus examine now the intensity of this field, its spatial variation, and how it alters the NMR spectrum. We are interested in two configurations of the NMR sample and of the rotating conductor:

(1) The sample is placed inside a hollow conductor and rotates with it. This is the situation encountered in MACS. Although the solenoidal coil used for this type of experiments does not have cylindrical symmetry it can be replaced for the purpose of calculating the effects of eddy currents by a stack of planar circular loops. If the pitch of the coil is small, the small tilting of the wire should not modify significantly the current trajectories, which will remain very close to those displayed in Fig. 5(b). If

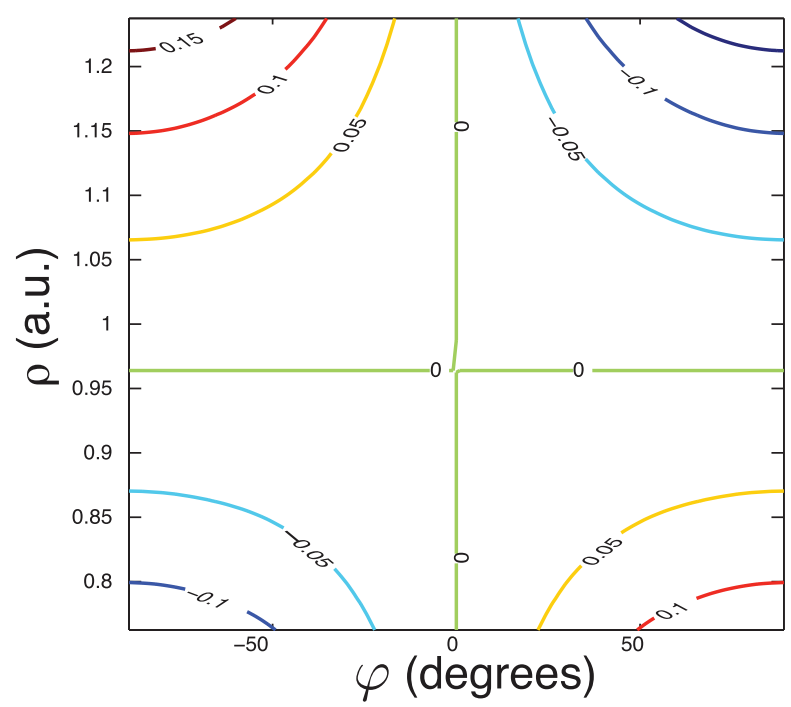

FIG. 6. Map of $\mathrm{J}_{\mathrm{Z}}$ in the xy half-plane $(\mathrm{x}>0)$ for the two cases displayed in Fig. 5. 


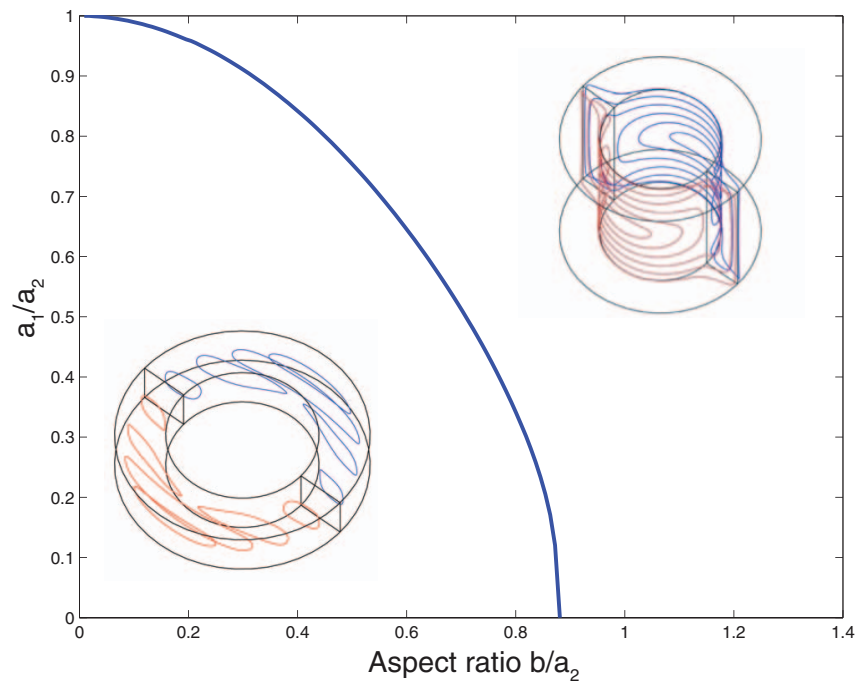

FIG. 7. Plot showing the domains of existence of the two topologies of current lines: on the right hand side of the plot the current lines are similar to those of a plain cylinder. On the left hand side a new type of trajectory appears as shown in the inset.

the coil is made by metal deposition it will usually have a rectangular cross section, and our formula will apply. If the coil is made from a wire of circular cross section, one crude approximation is to consider that the coil is equivalent to a coil made with a wire having a square cross section of the same area. A case to be mentioned is intentional heating of the sample by a surrounding conductive material. ${ }^{1}$ Various geometries of the conductor can be considered with the constraint that the rf field must not be shielded by the conductor. A metallic cylinder does not meet this requirement unless it is very thin. If this is not the case one has to introduce cuts; if the cuts are vertical our treatment does not apply, but it does, at least approximately for a helicoidal cut.

(2) A second configuration is when the sample is the conductive material itself and is studied by MAS-NMR (Ref. 1). The shape of the sample placed inside the MAS rotor is close to a cylinder. In this case also our formulae can be applied for the computation of the induced magnetic field.

\section{A. Angular variation of the fields generated by eddy currents}

Since the expressions for eddy currents are complex, one might expect a very complex spatial variation of $\vec{B}_{f}$ itself. It turns out, however, that in the frame $\mathrm{x}, \mathrm{y}, \mathrm{z}$ defined in Fig. 1, the three components of $\vec{B}_{f}: \mathrm{B}_{\mathrm{fx}}, \mathrm{B}_{\mathrm{fy}}$, and $\mathrm{B}_{\mathrm{fz}}$, have remarkably simple variations as a function of the azimuthal angle $\varphi$. They can be written (see Appendix B) as

$$
\begin{aligned}
B_{f x} & =h_{1}(\rho, z)+h_{2}(\rho, z) \cos (2 \varphi), \\
B_{f y} & =h_{2}(\rho, z) \sin (2 \varphi), \\
B_{f z} & =g_{3}(\rho, z) \cos (\varphi) .
\end{aligned}
$$
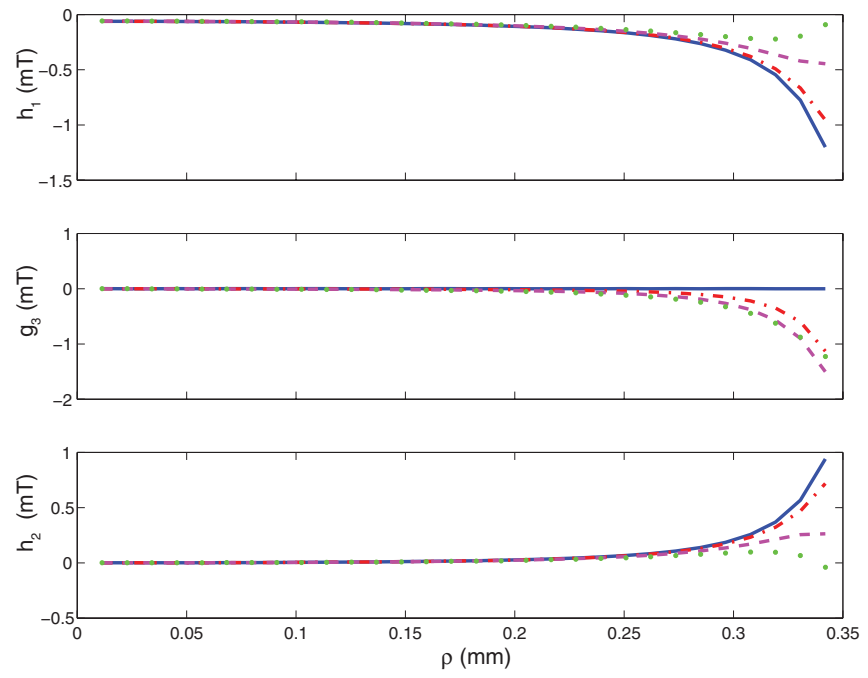

FIG. 8. Field coefficients $\mathrm{h}_{1}, \mathrm{~h}_{2}$, and $\mathrm{g}_{3}$ plotted as a function of $\rho$ for a copper loop of dimensions $\mathrm{a}_{1}=0.36 \mathrm{~mm}, \mathrm{a}_{2}=0.41 \mathrm{~mm}, \mathrm{~b}=0.025 \mathrm{~mm}$ spun at magic angle, at frequency $v_{\mathrm{r}}=10 \mathrm{kHz}$ in a field $\mathrm{B}_{0}=11.7 \mathrm{~T}$. Symbols used: $\mathrm{z}=0$ (blue -), $\mathrm{z}=0.5 \mathrm{~b}$ (red -.), $\mathrm{z}=\mathrm{b}$ (magenta - -), $\mathrm{z}=1.5 \mathrm{~b}$ (green ..).

The field coefficients $\mathrm{h}_{1}, \mathrm{~h}_{2}$, and $\mathrm{g}_{3}$ are proportional to $\sigma B_{0} \omega_{r} \sin (\theta)$. As a consequence of the known angular variation of $\vec{B}_{f}$ one just needs to compute the values of $\mathrm{h}_{1}, \mathrm{~h}_{2}$, and $\mathrm{g}_{3}$ for a set of $\rho$ and $\mathrm{z}$ values representative of the volume where one wants to evaluate the effects of $\vec{B}_{f}$.

\section{B. Case of a sample placed inside a hollow conductor}

\section{Spatial variation of field}

We consider here the field created by a single turn coil in order to visualize the intensity and location of $\vec{B}_{f}$ in this simple case. The case of a multi-turn coil simply requires adding the fields from the individual turns. A realistic example of a multi-turn coil will be examined in Sec. VI B 2.

For the calculation of the field coefficients $h_{1}, h_{2}$, and $\mathrm{g}_{3}$, we use Biot and Savart's law applied to $\vec{J}$ as given by Eqs. (8a)-(8c). For each value of the pair $\rho$, z, we compute $\vec{B}_{f}(\varphi=0)$ and $\vec{B}_{f}(\varphi=\pi / 4)$. The field coefficients $h_{1}(\rho, z)$, $h_{2}(\rho, z)$, and $g_{3}(\rho, z)$ are deduced from these two values. The ring considered for calculation is made from copper and its dimensions are $\mathrm{a}_{1}+\mathrm{a}_{2}=0.77 \mathrm{~mm}, \mathrm{a}_{2}-\mathrm{a}_{1}=0.05 \mathrm{~mm}$, and $b=0.025 \mathrm{~mm}$. The variation with $\rho$ of the three coefficients $h_{1}, h_{2}$, and $g_{3}$ is shown in Fig. 8. Four values of $z(0$, $\mathrm{b} / 2, \mathrm{~b}, 3 \mathrm{~b} / 2$ ) have been considered and a rotation frequency $v_{\mathrm{r}}$ $=10 \mathrm{kHz}$ is assumed.

From Fig. 8 it can be seen that $\vec{B}_{f}$ is mainly located in the vicinity of the loop. For a small cross section loop, numerical calculations show that as for the power dissipation (see Eq. (13)), the field parameters $h_{1}, g_{2}$, and $g_{3}$ have very different dependences upon the side lengths $b$ and $a_{2}-a_{1}$ of the cross section. They increase much more rapidly with $b$ than with $\mathrm{a}_{2}-\mathrm{a}_{1}$. As already mentioned, they are proportional to $B_{0}$ and to the rotation angular velocity, as do the eddy currents from which they originate. 


\section{Influence on the NMR spectrum}

The NMR frequency is sensitive only to the projection $\mathrm{B}_{\mathrm{f}}{ }^{\prime}$ of $\vec{B}_{f}$ on the direction of the applied magnetic field $\vec{B}_{0}$. Spinning of the sample produces a harmonic modulation of the field $\mathrm{B}_{\mathrm{f}}^{\prime}$ seen by one nucleus and thus of its resonance frequency. The problem is quite analogous to MAS-NMR of a powder sample with chemical shift anisotropy ${ }^{19}$ (CSA) where it is known that such a modulation gives rise to sidebands at frequencies $+/-\mathrm{N} v_{\mathrm{r}}$ from the nuclear Larmor frequency $\nu_{\mathrm{I}}=\omega_{\mathrm{I}} / 2 \pi=\gamma \mathrm{B}_{0} / 2 \pi$, where $\gamma$ is the gyromagnetic ratio of the nuclei.

Assuming $\vec{B}_{0}$ applied at the magic angle $\theta_{\mathrm{m}}=54.7^{\circ}$, the resonance of a nucleus is shifted by an amount

$$
\delta \omega_{I}(t)=\gamma B_{f}^{\prime}(t)=-\sin \left(\theta_{m}\right) \gamma B_{f y}(t)+\cos \left(\theta_{m}\right) \gamma B_{f z}(t),
$$

that is,

$$
\begin{aligned}
\delta \omega_{I}(t)= & -\sin \left(\theta_{m}\right) \gamma h_{2}(\rho, z) \sin (2 \varphi(t)) \\
& +\cos \left(\theta_{m}\right) \gamma g_{3}(\rho, z) \cos (\varphi(t)) .
\end{aligned}
$$

During rotation $\varphi$, whose initial value for a particular spin is denoted $\eta$, varies as $\cos \left(\omega_{\mathrm{r}} \mathrm{t}+\eta\right)$. We assume that the sample has cylindrical symmetry so that all values of $\eta$ between 0 and $2 \pi$ are represented with equal weight. $\delta \omega_{I}(t)$ can be written as

$$
\delta \omega_{I}(t)=C_{1} \cos \left(\omega_{r} t+\eta\right)+S_{2} \sin \left(2 \omega_{r} t+2 \eta\right)
$$

with

$$
\begin{aligned}
C_{1}(\rho, z) & =\gamma \cos \left(\theta_{m}\right) g_{3}(\rho, z) \\
\text { and } \quad S_{2}(\rho, z) & =-\gamma \sin \left(\theta_{m}\right) h_{2}(\rho, z) .
\end{aligned}
$$

We have used the same notation as in Ref. 19 and following the same lines as in Ref. 19, we derive the expression for the intensity $I_{N}=F_{N} F_{N} *$ of the sideband at distance $N v_{r}$ from the Larmor frequency. $\mathrm{F}_{\mathrm{N}}$ is given by ${ }^{19}$

$$
F_{N}=\frac{1}{2 \pi} \int_{0}^{2 \pi} e^{i\left[-N \Theta+\frac{C_{1}}{\omega_{r}} \sin (\Theta)-\frac{S_{2}}{2 \omega_{r}} \cos (2 \Theta)\right]} d \Theta,
$$

and can be easily computed with a modern mathematical software. Although there is a great similarity with the problem of chemical shift anisotropy, two differences should be outlined:

- the time variation of $\delta \omega_{\mathrm{I}}(\mathrm{t})$ contains only a $\cos \left(\omega_{\mathrm{r}} \mathrm{t}\right.$ $+\eta)$ and a $\sin \left(2 \omega_{\mathrm{r}} \mathrm{t}+2 \eta\right)$ term and no $\sin \left(\omega_{\mathrm{r}} \mathrm{t}+\eta\right)$ and $\cos \left(2 \omega_{\mathrm{r}} \mathrm{t}+2 \eta\right)$ as in the general CSA case. As a consequence the $+\mathrm{N}$ and $-\mathrm{N}$ sidebands have equal intensity.

- $\mathrm{C}_{1}$ and $\mathrm{S}_{2}$ are proportional to $\omega_{\mathrm{r}}$ so that $\mathrm{F}_{\mathrm{n}}$ does not depend on $\omega_{\mathrm{r}}$. This gives a convenient means to distinguish sidebands created by eddy currents from those originating from CSA or other anisotropy sources. It also means that if sidebands are present they cannot be removed by an increase of the spinning frequency. They can only be reduced by an adequate dimensioning of the coil; for example, one can reduce the cross section of the wire and increase the number of turns when this does not lead to excessive inductance of

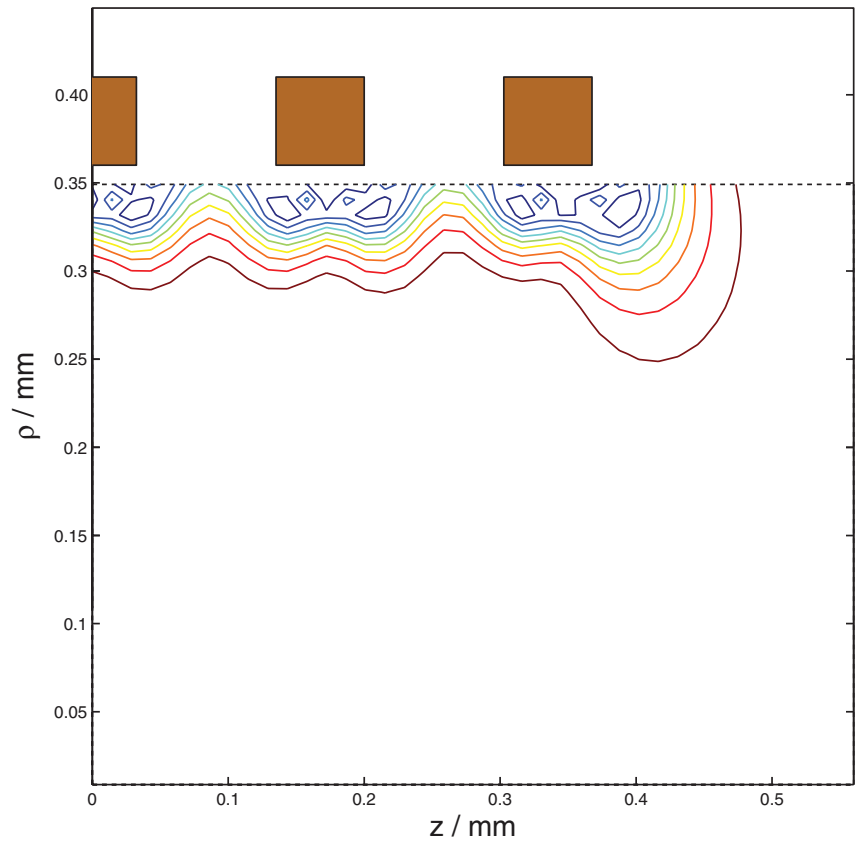

FIG. 9. Contour lines of the central peak intensity $I_{0}$ of the NMR line inside a 5 turn coil, modeled as a five-ring stack. Only the first quadrant of the $\rho$-z plane has been represented; the other ones are obtained by symmetry about the $\rho$ and z-axis. The internal diameter of the coil is $\varphi=0.72 \mathrm{~mm}$ and its overall length $0.75 \mathrm{~mm}$. The 9 levels of the contour lines going toward the axis of the coil are equally spaced between 0.1 and 0.9 . The wire cross sections are shown as square brown blocks.

the coil; if the number of turns cannot be increased, one can in the case of a rectangular cross-section play on the values of $b$ and $a_{2}-a_{1}$. From the results of Sec. VI B 1 obtained for a single turn coil, one can predict that a reduction of the length $b$ and to a lesser extent of width $a_{2}-a_{1}$ will decrease the intensities of the sidebands; it will also increase the rf resistance of the coil in a non-trivial way that should be correctly appreciated. $^{20}$

For high resolution MACS experiments it is important that sidebands are kept at a negligible level. This can be checked by mapping the intensity of the central peak $(\mathrm{N}=0)$ inside the coil. We performed this calculation for a coil suitable for proton MACS experiments at $500 \mathrm{MHz}$. The characteristics of this coil are: 5 turns, loop separation: $0.175 \mathrm{~mm}$, loop dimensions: $\mathrm{a}_{1}=0.36 \mathrm{~mm}, \mathrm{a}_{2}=0.41 \mathrm{~mm}$, $\mathrm{b}=0.025 \mathrm{~mm}$.

The results (independent of $v_{r}$ ) are shown in Fig. 9. For a cylindrical sample of diameter $=0.58 \mathrm{~mm}$ and length $0.62 \mathrm{~mm}$, the central peak intensity is everywhere higher than 0.9 . The filling factor of such a sample with respect to the internal volume of the coil is $60 \%$; this dimensioning of coil and sample would be suitable for high resolution magic angle coil spinning experiments.

\section{Field inside a plain cylinder}

The calculation of the induced field $\vec{B}_{f}$ at a point $\mathrm{M}$ inside the conductor requires some care because one needs to evaluate an integral that contains in the case of the vector 

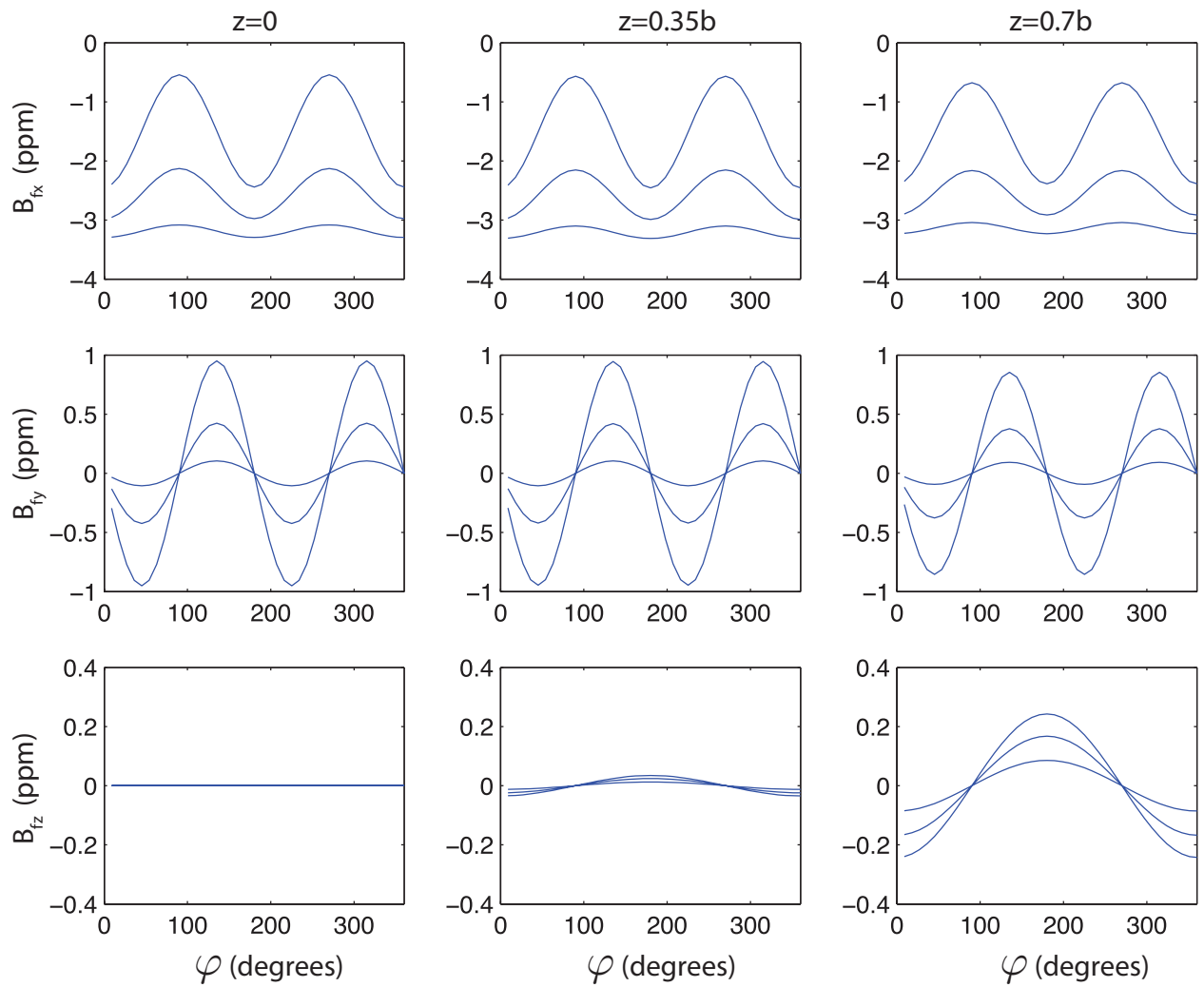

FIG. 10. Angular variation of the 3 components $\mathrm{B}_{\mathrm{fx}}, \mathrm{B}_{\mathrm{fy}}$, and $\mathrm{B}_{\mathrm{fz}}$ expressed in ppm of $\mathrm{B}_{0}$ (1st, 2nd, and 3rd row) of the field induced by eddy currents in a cylinder of diameter $=2.9 \mathrm{~mm}$, length $=2 \mathrm{~b}=14 \mathrm{~mm}$. Conductivity is $83 \mathrm{~S} / \mathrm{m}$ and rotation frequency $v_{\mathrm{r}}=10 \mathrm{kHz}$. Three altitudes $\mathrm{z}=0, \mathrm{z}=0.35 \mathrm{~b}$, and $\mathrm{z}=0.7 \mathrm{~b}$ have been considered (1st, 2 nd, and 3 rd columns). On each plot the data for 3 radii $(\mathrm{a} / 4, \mathrm{a} / 2$, and $3 \mathrm{a} / 4)$ are superimposed. The highest amplitude modulation corresponds to the largest radii.

potential $\vec{A}$ an 1/PM term (P is the running point inside the conductor, see Appendix B) that can cause divergences in numerical calculations. However, it is easily shown that the integral is absolutely convergent. The same is true for the Biot and Savart integral that gives $\vec{B}_{f}$. Our procedure to insure convergence is the following: we discretize the current distribution at points $\rho=(\mathrm{p}+1 / 2) \mathrm{d} \rho, \varphi=(\mathrm{q}+1 / 2) \mathrm{d} \varphi, \mathrm{z}$ $=(\mathrm{r}+1 / 2) \mathrm{dz}$ (p, q, r integers), and we compute the field at the points of coordinates $\rho=\operatorname{pd} \rho, \varphi=\operatorname{qd} \varphi, \mathrm{z}=\operatorname{rdz}$ by the Biot and Savart formula. We then scale down the values $\mathrm{d} \rho$, $\mathrm{d} \varphi, \mathrm{dz}$ of the mesh size. The computed values of $\vec{B}_{f}$ converge toward the exact value.

As a case of interest we consider again the experiment of Yesinowski et al. ${ }^{1}$ on a rotating CuI cylindrical sample of dimensions: diameter $=2.9 \mathrm{~mm}$ and length $=14 \mathrm{~mm}$. We take for the conductivity the value $\sigma=83 \mathrm{~S} / \mathrm{m}$, which corresponds to one of the samples studied in which important effects due to eddy currents were observed. The spectra were recorded in a field of $11.7 \mathrm{~T}$ on a sample spun at magic angle at spinning frequencies ranging between 0 and $10 \mathrm{kHz}$.

The values of $\mathrm{B}_{\mathrm{fx}}, \mathrm{B}_{\mathrm{fy}}$, and $\mathrm{B}_{\mathrm{fz}}$ are displayed in Fig. 10 for three values of $\rho$ and three altitudes $\mathrm{z}$. A rotation frequency $v_{\mathrm{r}}=10 \mathrm{kHz}$ is assumed and the results are given in ppm since $\vec{B}_{f}$ is proportional to $\mathrm{B}_{0}$. The angular variations displayed in Fig. 10 are in agreement with Eq. (16); this can be considered as a test of consistency of our calculation.

The resonance frequency inside the conductor given by Eq. (17) is spread by an amount of the order of the amplitude of variation of $\mathrm{B}_{\mathrm{fy}}$ and $\mathrm{B}_{\mathrm{fz}}$, i.e., about $1 \mathrm{ppm}$ at $v_{\mathrm{r}}$ $=10 \mathrm{kHz}$. This is an order of magnitude less than broadening expected from temperature gradients originating from eddy current heating. The data from Ref. 1 indicate a temperature gradient of $40 \mathrm{~K}$ at $v_{\mathrm{r}}=10 \mathrm{kHz}$ which translates for $\mathrm{CuI}$ into a broadening of $10 \mathrm{ppm}$. Furthermore, in the case of the induced fields effects, the resonance frequency shifts should be efficiently averaged by magic angle rotation and give rise to very small rotational sidebands. Indeed for copper nuclei at $11.7 \mathrm{~T}$ a $1 \mathrm{ppm}$ spread corresponds to $0.13 \mathrm{kHz}$ which is small compared to the assumed rotation frequency $v_{\mathrm{r}}=10 \mathrm{kHz}$. For this reason we did not reproduce the calculation developed in Sec. VI B 2 since it is expected to predict a negligible effect on the intensity of the central peak's intensity.

In conclusion we have shown, in this case at least, that the fields created by eddy currents inside a conductive sample have only a small effect on the MR spectrum. Samples with higher conductivity would exhibit larger induced fields but they would also be very difficult to spin. One would furthermore be faced with the problem of rf penetration inside the sample since even 
with $\sigma=83 \mathrm{~S} / \mathrm{m}$ the skin depth at $100 \mathrm{MHz}$ is equal to $\delta=5.5 \mathrm{~mm}$.

\section{CONCLUSION}

Spinning conductive samples is a very common case in MR studies and the effects of eddy currents are often taken into account in a qualitative manner, by claiming a temperature increase and degradation of line-width. Since the case of coil spinning has also become increasingly important, an explicit treatment of such effects seemed necessary, in order to establish a good understanding of the phenomena and to obtain quantitative formulae that allow for experiment optimization.

Here, we have established exact analytical solutions for the 3D problem of a plain or hollow cylinder spinning at an angle inside an ultra-uniform strong magnetic field. The results allow us to calculate the power dissipation and the current trajectories, and thus the additional magnetic field generated by these currents. Our results (in particular, the current lines) being exact could serve as benchmarks for 3D numerical software. Furthermore, they can be used for obtaining easily the power dissipation levels without the use of software, for the case of conductive samples, and they provide information also about the magnetic field distortions and inhomogeneities that will be present in the NMR spectrum. Our analysis takes into account also the presence of a coil spinning and predicts extremely small eddy current effects on the NMR spectra of samples spinning at the magic angle and detected by a spinning coil detector. Such effects are difficult to be observed in common experiments, and here we have chosen the appropriate conditions in order to observe these effects and validate further our claims.

We believe our analysis could be not only used for improving spinning samples, spinning detectors, or even spinning magnets for magnetic resonance but also for analyzing the MR response of conductive samples of technological interest such as battery materials, ionic conductors, or of biological interest such as bio-solids or tissues.

\section{ACKNOWLEDGMENTS}

The authors would like to thank Dr. C. Hugon and Dr. J. P. Yesinowski for stimulating discussions. This work was supported by the European Research Council under the European Community's Seventh Framework Programme (FP7/20072013): ERC Grant Agreement No. 205119.

\section{APPENDIX A: APPROXIMATE FORMULAE FOR CASES OF PARTICULAR GEOMETRIES}

As in Sec. IV B we define $\varepsilon$ and $\beta$ by

$$
\begin{aligned}
& a_{1}=(1-\varepsilon / 2) a, \\
& a_{2}=(1+\varepsilon / 2) a, \\
& b=\beta a,
\end{aligned}
$$

and examine in turn the cases $\varepsilon, \beta \ll 1, \beta=\infty, \varepsilon \ll 1$ and $\beta$ finite, and $\beta \ll 1$ and $\varepsilon$ finite.

\section{Small cross section toroid $(\varepsilon, \beta \ll 1)$}

A more extended development of $\mathrm{P}$ than the one given in Sec. IV B yields

$$
\begin{aligned}
\frac{P}{\sigma\left(B_{0} \omega_{r} \sin (\theta)\right)^{2}}= & \frac{4 \pi}{3} a^{5} \beta^{3} \varepsilon-\pi a^{5} \beta^{4} \sum_{k} \frac{1}{k^{5}} \\
& \times\left[\left(8-\frac{\beta^{2}}{k^{2}}+\ldots\right) \tanh \left(\frac{1}{2} k \frac{\varepsilon}{\beta}\right)\right. \\
& \left.+\frac{7}{2} \frac{\beta \varepsilon}{k \cosh ^{2}\left(\frac{1}{2} k \frac{\varepsilon}{\beta}\right)}+\frac{\varepsilon^{2}}{\sinh \left(k \frac{\varepsilon}{\beta}\right)}+\ldots\right], \\
k= & (2 n+1) \frac{\pi}{2}, n=0,1, \ldots
\end{aligned}
$$

\section{Infinitely long tube $(\beta=\infty)$}

In this case it is convenient to use for the exciting field the form $\vec{E}^{\prime}=-B_{0} \omega_{r} \sin (\theta) y \vec{u}_{z}=-B_{0} \omega_{r} \sin (\theta) \rho \sin (\varphi) \vec{u}_{z}$.

This field produces surfaces charges at $\mathrm{z}= \pm \infty$ that can be neglected. We can write directly

$$
\vec{J}=\sigma \vec{E}^{\prime}=-\sigma B_{0} \omega_{r} \sin (\theta) \rho \sin (\varphi) \vec{u}_{z} .
$$

The power dissipated per unit length is straightforwardly deduced as

$$
\frac{1}{\sigma\left(B_{0} \omega_{r} \sin (\theta)\right)^{2}} \frac{d P}{d z}=\frac{\pi}{4}\left(a_{2}^{4}-a_{1}^{4}\right)
$$

It is known ${ }^{5}$ that a cylindrical sheet of current flowing in the $\mathrm{z}$ direction with a $\sin (\varphi)$ azimuthal variation produces a homogeneous internal field pointing in the $\mathrm{x}$ direction. In the present case integration of the fields produced by the cylindrical layers of current between $\mathrm{a}_{1}$ and $\mathrm{a}_{2}$ yields a uniform field of value

$$
B_{x}=-\frac{\mu_{0}}{4} \omega_{r} \sigma B_{0} \sin (\theta)\left(a_{2}^{2}-a_{1}^{2}\right)=-B_{0} \sin (\theta) \frac{\left(a_{2}^{2}-a_{1}^{2}\right)}{2 \delta^{2}} .
$$

\section{Very thin tube of finite length $(\varepsilon \ll 1$ and $\beta$ finite)}

Using the approach described in supplemental material, ${ }^{13}$ one can derive approximate values of $\mathrm{m}$, of the coefficients $A_{m}$ and $B_{m}$, and of the corresponding value of P. The calculation will not be reproduced here. The final result to lowest order is

$$
\frac{P}{\sigma\left(B_{0} \omega_{r} \sin (\theta)\right)^{2}}=2 \pi a^{4} \varepsilon(b-a \tanh (b / a)) .
$$

Formulae (A2) and (A3) agree in the limit $\mathrm{a}_{2}-\mathrm{a}_{1} \rightarrow 0$ and $\mathrm{b}$ $\rightarrow \infty$. 


\section{Very thin annular disk ( $\beta \ll 1$ and $\varepsilon$ finite)}

For small values of $b / a_{2}$ one can show that the development of $\mathrm{P}$ is

$$
\begin{aligned}
& \frac{P}{\sigma\left(B_{0} \omega_{r} \sin (\theta)\right)^{2}} \\
& =\frac{2 \pi}{3} b^{3}\left(a_{2}^{2}-a_{1}^{2}\right)-\pi \sum_{k}\left\{\frac { 4 b ^ { 4 } } { k ^ { 5 } \operatorname { s i n h } ( u ) } \left[\left(a_{2}+a_{1}\right) \cosh (u)\right.\right. \\
& \left.\quad-2 \sqrt{a_{1} a_{2}}\right]+\frac{7 b^{5}}{k^{6} \sinh ^{2}(u)} \frac{a_{2}-a_{1}}{a_{1} a_{2}} \\
& \left.\quad \times\left(\sqrt{a_{1} a_{2}} \cosh (u)-\frac{a_{2}+a_{1}}{2}\right)+\ldots\right\},
\end{aligned}
$$

where

$$
\begin{aligned}
& u=k \frac{a_{2}-a_{1}}{b}, \\
& k=\frac{(2 n+1) \pi}{2 b}, n=0,1, \ldots .
\end{aligned}
$$

Development (A1) is recovered in the limit $\mathrm{a}_{1} \rightarrow \mathrm{a}_{2}$.

\section{APPENDIX B: ANGULAR VARIATION OF THE FIELD GENERATED BY EDDY CURRENTS}

The symmetry properties of the field induced by eddy currents are related to those of the vector potential $\vec{A}_{f}$ whose expression is

$$
\vec{A}_{f}=\frac{\mu_{0}}{4 \pi} \int_{a_{1}}^{a_{2}} \int_{-\pi}^{\pi} \int_{-b}^{b} \frac{\vec{J}(\rho, \varphi, z)}{|P M|} \rho d \rho d \varphi d z,
$$

where $\mathbf{M}\left(\rho_{0}, \theta_{0}, \varphi_{0}\right)$ is the point where $\vec{A}_{f}$ is calculated and integration is performed on the coordinates $(\rho, \theta, \varphi)$ of the running point $\mathrm{P}$ inside the current distribution.

\section{Case of a hollow conductor}

If condition $\rho_{0}<\rho$ holds for all points $\mathrm{P}$ inside the conductor we can develop 1/PM. Starting from

$$
\frac{1}{|P M|}=\frac{1}{\sqrt{\rho_{0}^{2}+\rho^{2}+\left(z-z_{0}\right)^{2}-2 \rho_{0} \rho \cos \left(\varphi-\varphi_{0}\right)}},
$$

we let

$$
\begin{aligned}
& \rho=c \sin \alpha, \\
& z-z_{0}=c \cos \alpha, \\
& \varphi-\varphi_{0}=\psi,
\end{aligned}
$$

which gives

$$
\begin{aligned}
\frac{1}{|P M|} & =\frac{1}{c \sqrt{1-2 \frac{\rho_{0}}{c} \sin \alpha \cos \psi+\left(\frac{\rho_{0}}{c}\right)^{2}}} \\
& =\frac{1}{c}\left[1+\sum_{n=1}^{\infty}\left(\frac{\rho_{0}}{c}\right)^{n} P_{n}(\sin \alpha \cos \psi)\right] .
\end{aligned}
$$

We have used the well known definition of Legendre polynomials $\mathrm{P}_{\mathrm{n}}(\mathrm{x})$ from the generating function $(1-2 \mathrm{xt}$ $\left.+\mathrm{t}^{2}\right)^{-1 / 2} \cdot P_{n}(\sin \alpha \cos \psi)$ itself can be developed using the addition theorem

$$
\begin{aligned}
P_{n}(\sin \alpha \cos \psi)= & P_{n}(0) P_{n}(\cos \alpha)+2 \sum_{m=1}^{n} \frac{(n-m) !}{(n+m) !} \\
& \times P_{n}^{m}(0) P_{n}^{m}(\cos \alpha) \cos (m \psi) .
\end{aligned}
$$

Using the fact that when $\mathrm{n}-\mathrm{m}$ is odd $P_{n}^{m}(0)=0$ and when $\mathrm{n}-\mathrm{m}$ is even $P_{n}^{m}(0)=(-1)^{\frac{n-m}{2}} \frac{(n+m-1) ! !}{(n-m) ! !}$, we obtain

$$
\begin{aligned}
\frac{1}{|P M|}= & \frac{1}{c}+2 \sum_{p=0}^{\infty} \frac{(-1)^{p} \rho_{0}^{2 p+1}}{c^{2 p+2}} \sum_{q=0}^{p}(-1)^{q} \frac{(2 p-2 q-1) ! !}{(2 p+2 q+2) ! !} \\
& \times P_{2 p+1}^{2 q+1}(\cos \alpha) \cos (2 q+1) \psi \\
& +\sum_{p=1}^{\infty} \frac{(-1)^{p} \rho_{0}^{2 p}}{c^{2 p+1}}\left[\frac{(2 p-1) ! !}{(2 p) ! !} P_{2 p}(\cos \alpha)\right. \\
& \left.+2 \sum_{q=1}^{p}(-1)^{q} \frac{(2 p-2 q-1) ! !}{(2 p+2 q) ! !} P_{2 p}^{2 q}(\cos \alpha) \cos (2 q \psi)\right] .
\end{aligned}
$$

The components of $\vec{J}$ are proportional either to $\sin \varphi$ or to $\cos \varphi$. The integrals to be considered in the calculation of $\vec{A}_{f}$ are thus $\int_{-\pi}^{\pi} \sin \varphi \cos m\left(\varphi-\varphi_{0}\right) d \varphi$ and $\int_{-\pi}^{\pi} \cos \varphi \cos m\left(\varphi-\varphi_{0}\right) d \varphi$; they are equal to zero except for $\mathrm{m}=1$ where one has $\int_{-\pi}^{\pi} \sin \varphi \cos \left(\varphi-\varphi_{0}\right) d \varphi=\pi \sin \varphi_{0}$ and $\int_{-\pi}^{\pi} \cos \varphi \cos \left(\varphi-\varphi_{0}\right) d \varphi=\pi \cos \varphi_{0}$. In the development (B1), one can just keep the terms with $\mathrm{q}=0$, that is, $2 \sum_{p=0}^{\infty} \frac{(-1)^{p} \rho^{2 p+1}}{c^{2 p+2}} \frac{(2 p-1) ! !}{(2 p+2) ! !} P_{2 p+1}^{1}(\cos \alpha) \cos \psi$; this leads to components of $\vec{A}_{f}$ of the form

$$
\begin{aligned}
& A_{\rho}=f_{1}\left(\rho_{0}, z_{0}\right) \sin \left(\varphi_{0}\right), \\
& A_{\varphi}=f_{2}\left(\rho_{0}, z_{0}\right) \cos \left(\varphi_{0}\right), \\
& A_{z}=f_{3}\left(\rho_{0}, z_{0}\right) \sin \left(\varphi_{0}\right) .
\end{aligned}
$$

In cylindrical coordinates the components of $\vec{B}_{f}=\vec{\nabla}$ $\times \vec{A}_{f}$ are of the form

$$
\begin{aligned}
& B_{\rho}=g_{1}\left(\rho_{0}, z_{0}\right) \cos \left(\varphi_{0}\right), \\
& B_{\varphi}=g_{2}\left(\rho_{0}, z_{0}\right) \sin \left(\varphi_{0}\right), \\
& B_{z}=g_{3}\left(\rho_{0}, z_{0}\right) \cos \left(\varphi_{0}\right),
\end{aligned}
$$

which yield for the Cartesian components of $\vec{B}_{f}$ :

$$
\begin{aligned}
& B_{x}=h_{1}\left(\rho_{0}, z_{0}\right)+h_{2}\left(\rho_{0}, z_{0}\right) \cos \left(2 \varphi_{0}\right), \\
& B_{y}=h_{2}\left(\rho_{0}, z_{0}\right) \sin \left(2 \varphi_{0}\right), \\
& B_{z}=g_{3}\left(\rho_{0}, z_{0}\right) \cos \left(\varphi_{0}\right)
\end{aligned}
$$

with

$$
\begin{aligned}
& h_{1}\left(\rho_{0}, z_{0}\right)=\frac{1}{2}\left[g_{1}\left(\rho_{0}, z_{0}\right)-g_{2}\left(\rho_{0}, z_{0}\right)\right], \\
& h_{2}\left(\rho_{0}, z_{0}\right)=\frac{1}{2}\left[g_{1}\left(\rho_{0}, z_{0}\right)+g_{2}\left(\rho_{0}, z_{0}\right)\right] .
\end{aligned}
$$




\section{General case}

If point $\mathrm{M}$ lies inside the conductor, we can decompose the latter in two regions: one for which $\rho_{0}<\rho$ and the other for which $\rho_{0}>\rho$. For this region one can make a development in $\rho / \rho_{0}$. The same angular variation is found for this second region. Equation (B2) is thus valid in the general case.

${ }^{1}$ J. P. Yesinowski, H. D. Ladouceur, A. P. Purdy, and J. B. Miller, "Electrical and ionic conductivity effects on magic-angle spinning nuclear magnetic resonance parameters of CuI," J. Chem. Phys. 133, 234509 (2010).

${ }^{2}$ D. Sakellariou, G. Le Goff, and J.-F. Jacquinot, "High resolution, high sensitivity NMR of nanoliter anisotropic samples by coil spinning," Nature (London) 447, 694-697 (2007).

${ }^{3}$ P. M. Aguiar, J.-F. Jacquinot, and D. Sakellariou, "Experimental and numerical examination of eddy (Foucault) currents in rotating micro-coils: Generation of heat and its impact on sample temperatures," J. Magn. Reson. 200, 6-14 (2009).

${ }^{4}$ A. Trakic, B. K. Li, E. Weber, H. Wang, S. Wilson, and S. Crozier, "A rapidly rotating RF coil for MRI," Concepts Magn. Reson. 35B, 59-66 (2009).

${ }^{5}$ W. R. Smythe, Static and Dynamic Electricity (McGraw Hill, New York, 1968).

${ }^{6}$ J. Lameraner and M. Stafl, Eddy Currents (Illife Books, London, 1966).

${ }^{7}$ A. D. Abramov, "Eddy current losses in a rotating magnetic field," Russ. Phys. J. 14, 25-28 (1971).

${ }^{8}$ R. Schäfer and C. Heiden, "Eddy current losses of cylindrical conductors rotating in a magnetic field," Appl. Phys. 9, 121-125 (1976).
${ }^{9}$ D. J. Groh and J. R. Dixon, Jr., "Torque induced on a conducting cylinder by a slowly rotating magnetic field," J. Appl. Phys. 54, 1135-1136 (1983).

${ }^{10}$ L. Landau, E. Lifchitz, and L. P. Pitaevskii, Electrodynamics of Continuous Media (Pergamon, Oxford, 1960).

${ }^{11}$ J. D. Jackson, Classical Electrodynamics, 3rd ed. (Wiley, New York, 1998).

${ }^{12}$ G. Arfken, Mathematical Methods for Physicists, 2nd ed. (Academic, New York, 1970).

${ }^{13}$ See supplementary material at http://dx.doi.org/10.1063/1.4756948 for a derivation of formulae obtained in previous works and for direct integration of Eq. (10).

${ }^{14}$ G. Meunier, J. C. Sabonnadiere, and J. L. Coulomb, "The finite element post-processor of FLUX3D (field computation package)," IEEE Trans. Magn. 27, 3786 (1991).

${ }^{15}$ T. L. Peck, R. L. Magin, and P. C. Lauterbur, "Design and analysis of microcoils for NMR microscopy,” J. Magn. Reson. B 108, 114 (1995).

${ }^{16} \mathrm{R}$. A. Wind and J. Z. Hu, "In vivo and ex vivo high-resolution ${ }^{1} \mathrm{H}$ NMR in biological systems using low-speed magic angle spinning," Prog. Nucl. Magn. Reson. Spectrosc. 49, 207 (2006).

${ }^{17}$ A. Wong, P. M. Aguiar, and D. Sakellariou, "Slow magic-angle coil spinning: A high-sensitivity and high-resolution NMR strategy for microscopic biological specimens," Magn. Reson. Med. 63, 269 (2010).

${ }^{18} \mathrm{D}$. S. Wishart et al., " ${ }^{11} \mathrm{H},{ }^{13} \mathrm{C}$ and ${ }^{15} \mathrm{~N}$ chemical shift referencing in biomolecular NMR," J. Biomol. NMR 6, 135 (1995).

${ }^{19}$ M. Mehring, Principles of High Resolution NMR in Solids (SpringerVerlag, 1983).

${ }^{20}$ J.-F. Jacquinot and D. Sakellariou, "NMR signal detection using inductive coupling: Applications to rotating microcoils," Concepts Magn. Reson. 38A, 33-51 (2011), and references therein. 